\title{
In Situ Metal-Ligand Reactions under Solvent-Dependent Hydro(solvo)thermal Conditions: Structures, Mass Spectrometry, and Magnetism
}

Ye Tao,${ }^{\dagger}$ Xu-Hong Pang,${ }^{\dagger}$ Hai-Ye Li,${ }^{\dagger}$ He-Dong Bian,,${ }^{*}+$ Han-Fu Liu,${ }^{\dagger}$ Fu-Ping Huang ${ }^{*}, \dagger$

†State Key Laboratory for the Chemistry and Molecular Engineering of Medicinal Resources, School of Chemistry and Pharmacy, Guangxi Normal University, Guilin 541004, P. R. China.

${ }^{\ddagger}$ Key Laboratory of Development and Application of Forest Chemicals of Guangxi, Guangxi University of Nationalities, Nanning 530006, P. R. China.

1 Experimental section

2 TGA curves of the complexes

3 Structures of complexes

4 SHAPE analysis of the Dy ${ }^{\mathrm{III}}$ ions

5 Ligand details

6 PXRD data of the complexes

7 ESI-MS of the complexes

8 Magnetism

9 Crystallographic data and selected bond lengths $[\AA]$ and angles $\left[{ }^{\circ}\right]$ for the complexes 


\section{Experimental Section}

\subsection{Experimental Materials}

The ligand of bis[3-(pyridin-2-yl)-1H-1,2,4-triazol-5-yl]methane $\left(\mathrm{H}_{2} \mathrm{~L}^{0}\right)$ was prepared according to a literature procedure. ${ }^{1,2}$ All reagents and solvents for synthesis and analysis were commercially available and used as received.

\subsection{Synthesis of the complexes 1-8 \\ $\left[\mathrm{Dy}\left(\mathrm{H}_{2} \mathrm{~L}^{0}\right)\left(\mathrm{NO}_{3}\right)\left(\mathrm{H}_{2} \mathrm{O}\right)_{3}\right] \cdot 2 \mathrm{NO}_{3} \cdot \mathrm{H}_{2} \mathrm{O}(1)$}

A mixture of $\mathrm{H}_{2} \mathrm{~L}^{0}(0.30 \mathrm{mmol}, 0.0912 \mathrm{~g})$, Dy $(\mathrm{NO})_{3} \cdot 6 \mathrm{H}_{2} \mathrm{O}(0.60 \mathrm{mmol}, 0.2740 \mathrm{~g})$ and $\mathrm{NaN}_{3}(0.60$ mmol, $0.0390 \mathrm{~g}$ ) were added in $15 \mathrm{~mL}$ EtOH. The mixture was stirred at room temperature for 20 min. The resulting reaction mixture was sealed in a Teflon-lined stainless steel vessel $(25 \mathrm{~mL})$ and maintained at $140{ }^{\circ} \mathrm{C}$ for 3 days, and then cooled slowly to room temperature to yield yellow single crystals suitable for X-ray diffraction. Yield: $45 \%$ [based on Dy ${ }^{\mathrm{III}}$ ]. IR $\left(\mathrm{KBr}, \mathrm{cm}^{-1}\right)$ : 3405(s), 3143(s), 3091(s), 3043(s), 2930(s), 2802(s), 1653(s), 1609(s), 1562(w), 1479(s), 1430(s), 1384(s), 1301(s), 1156(w), 1059(w), 1022(w), 914(w), 864(m), 798(m), 742(m), 636(w).

\section{$\left[\mathrm{Dy}\left(\mathrm{L}^{1}\right)(\mathrm{Ac})\left(\mathrm{H}_{2} \mathrm{O}\right)_{3}\right] \cdot 4.5 \mathrm{H}_{2} \mathrm{O}(2)$}

A mixture of $\mathrm{H}_{2} \mathrm{~L}^{0}(0.30 \mathrm{mmol}, 0.0912 \mathrm{~g}), \mathrm{Dy}(\mathrm{Ac})_{3} \cdot 4 \mathrm{H}_{2} \mathrm{O}(0.60 \mathrm{mmol}, 0.2686 \mathrm{~g})$ and $\mathrm{NaN}_{3}(0.60$ mmol, $0.0390 \mathrm{~g})$ were added in $\mathrm{H}_{2} \mathrm{O}-\mathrm{MeOH}(\mathrm{v} / \mathrm{v}=14: 1)$. The mixture was stirred at room temperature for $20 \mathrm{~min}$. The resulting reaction mixture was sealed in a Teflon-lined stainless steel vessel $(25 \mathrm{~mL})$ and maintained at $140{ }^{\circ} \mathrm{C}$ for 3 days, and then cooled slowly to room temperature and filtered. The filtrate was volatilized for one week and complex 2 was obtained. Yield: $15 \%$ [based on Dy ${ }^{\mathrm{III}}$ ]. IR (KBr, $\left.\mathrm{cm}^{-1}\right)$ : 3403(s), 3140(s), 3085(s), 3039(s), 2928(s), 2799(s), 1652(w), 1604(s), 1561(w), 1477(s), 1428(w), 1383(s), 1293(w), 1154(w), 1057(w), 1015(w), 913(w), 863(w), 797(m), 742(m), 629(w).

\section{$\left[\mathrm{Dy}\left(\mathrm{L}^{1}\right)\left(\mu_{2}-\mathrm{OH}\right)\right]_{\mathrm{n}} \cdot 3 \mathrm{H}_{2} \mathrm{O}(3)$ and $\left[\mathrm{Dy}_{2}\left(\mathrm{~L}^{2}\right)\left(\mathrm{NO}_{3}\right)_{2}\left(\mathrm{H}_{2} \mathrm{O}\right)_{6}\right] \cdot 2 \mathrm{H}_{2} \mathrm{O}(4)$}

A procedure similar to that for 2 was followed except that Dy(Ac) $)_{3} \cdot 4 \mathrm{H}_{2} \mathrm{O}$ was replaced by Dy $(\mathrm{NO})_{3} \cdot 6 \mathrm{H}_{2} \mathrm{O}$. When the reaction cools to room temperature, two types of single crystals were obtained (complexes 3 and 4). Two types of coordination complexes were carefully picked out and 
separated from each other by hand, on the basis of their different crystal shapes and/or cell determination.

(3). Yield: $32 \%$ [based on Dy ${ }^{\mathrm{II}}$ ]. IR (KBr, $\left.\mathrm{cm}^{-1}\right)$ : 3416(s), 1604(s), 1523(s), 1477(s), 1422(s), 1379(s), 1282(w), 1174(w), 1118(w), 1088(w), 1021(w), 927(s), 798(m), 753(m), 725(m), 636(w), 451(w).

(4). Yield: $17 \%$ [based on Dy $\left.{ }^{\mathrm{II}}\right]$. IR (KBr, $\left.\mathrm{cm}^{-1}\right)$ : 3136(s), 1606(s), 1470(s), 1384(s), 1320(s), 1287(s), 1174(w), 1090(w), 1025(w), 922(w), 797(m), 751(m), 723(m), 635(w).

\section{$\operatorname{Dy}\left(\mathrm{H}_{2} \mathbf{L}^{3}\right)\left(\mathrm{NO}_{3}\right)_{2}\left(\mathrm{H}_{2} \mathrm{O}\right)(5)$}

A procedure similar to that for $\mathbf{1}$ was followed except that EtOH was replaced by acetonitrile. Yield: $25 \%$ [based on Dy ${ }^{\mathrm{III}}$ ]. IR (KBr, cm $\left.{ }^{-1}\right): 3384(\mathrm{~s}), 3298(\mathrm{~s}), 1608(\mathrm{~s}), 1535(\mathrm{~s}), 1460(\mathrm{~s}), 1425(\mathrm{~s}), 1385(\mathrm{~s})$, 1271(w), 1179(w), 1106(w), 1014(w), 930(w), 802(m), 756(m), 721(m), 681(w), 638(m), 642(m).

\section{$\operatorname{Dy}\left(\mathrm{HL}^{4}\right)\left(\mathrm{NO}_{3}\right)_{3}(6)$}

A procedure similar to that for $\mathbf{1}$ was followed except that EtOH was replaced by acetylacetone. Yield: $55 \%$ [based on Dy ${ }^{\mathrm{III}}$ ]. IR (KBr, $\left.\mathrm{cm}^{-1}\right)$ : 3196(s), 2992(w), 1634(w), 1589(s), 1523(s), 1473(s), 1385(s), 1283(s), 1171(w), 1127(w), 1016(w), 916(w), 803(m), 746(m), 715(m), 636(w), 524(w).

\section{$\mathrm{Dy}_{4}\left(\mathrm{~L}^{5}\right)_{2}\left(\mu_{3}-\mathrm{OH}\right)_{2}(\mathrm{Ac})_{6}\left(\mathrm{NO}_{3}\right)_{2}(7)$}

A mixture of $\mathrm{H}_{2} \mathrm{~L}^{0}(0.30 \mathrm{mmol}, 0.0912 \mathrm{~g})$, Dy(NO) $)_{3} \cdot 6 \mathrm{H}_{2} \mathrm{O}(0.30 \mathrm{mmol}, 0.1370 \mathrm{~g})$, Dy $(\mathrm{Ac})_{3} \cdot 4 \mathrm{H}_{2} \mathrm{O}$ $(0.30 \mathrm{mmol}, 0.1235 \mathrm{~g}), \mathrm{NaN}_{3}(0.60 \mathrm{mmol}, 0.0390 \mathrm{~g})$ were added in $15 \mathrm{~mL}$ acetone. The mixture was stirred at room temperature for $20 \mathrm{~min}$. The resulting reaction mixture was sealed in a Teflonlined stainless steel vessel $(25 \mathrm{~mL})$ and maintained at $140{ }^{\circ} \mathrm{C}$ for 3 days, and then cooled slowly to room temperature to yield yellow single crystals suitable for X-ray diffraction. Yield: $58 \%$ [based on Dy $\mathrm{yII}_{\mathrm{III}}$. IR (KBr, $\left.\mathrm{cm}^{-1}\right)$ : 3433(s), 1571(s), 1439(s), 1384(s), 1155(w), 1089(w), 1021(w), 926(w), 667(w).

\section{$\mathrm{Dy}_{4}\left(\mathrm{~L}^{6}\right)_{2}\left(\mu_{3}-\mathrm{OH}\right)_{2}(\mathrm{Ac})_{6}\left(\mathrm{NO}_{3}\right)_{2}(8)$}

A procedure similar to that for 7 was followed except that acetone was replaced by 2-butanone. Yield: 47 \% [based on Dy ${ }^{\mathrm{III}}$ ]. IR (KBr, $\left.\mathrm{cm}^{-1}\right)$ : 3331(s), 1548(s), 1457(s), 1385(s), 1285(s), 1164(w), 
1056(w), 1016(w), 942(w), 799(m), 761(m), 723(m), 681(w).

Their thermogravimetric (TG) analyses are presented in Figures S1.

\subsection{Physical Measurements}

The magnetic susceptibility measurements of the polycrystalline samples were measured over the temperature range of 2-300 K with a Quantum Design MPMS-XL-7 SQUID magnetometer using an applied magnetic field of 1000 Oe. The TG analyses $\left(30-800{ }^{\circ} \mathrm{C}\right)$ for $\mathbf{1 - 8}$ were conducted on a PerkinElmer Diamond TG/DTA thermal analyzer in a flowing nitrogen atmosphere with a heating rate of $5{ }^{\circ} \mathrm{C} \mathrm{min}^{-1}$. The Fourier transform infrared data were recorded with PerkinElmer Spectrum One FT-IR spectrometer using $\mathrm{KBr}$ pellets in the wavenumber range of $400-4000 \mathrm{~cm}^{-1}$. Powder Xray diffraction $(\mathrm{PXRD})$ intensities were measured on a Rigaku D/max diffractometer equipped $(\mathrm{Cu}-$ $\mathrm{K} \alpha, \lambda=1.54056 \AA$ ). The single-crystalline powder samples were prepared by crushing the crystals and scanned from 3 to $50^{\circ}$ with a step of $5^{\circ} \mathrm{min}^{-1}$.

\subsection{X-Ray crystallography}

Single-crystal data of complexes 1-8 were collected on an Agilent SuperNova single-crystal diffractometer equipped with graphite monochromatic Mo-K $\alpha$ radiation $(\lambda=0.71073 \AA))$ at room temperature $(293 \mathrm{~K})$. Using Olex $2,{ }^{3}$ the structure was solved with the ShelXS ${ }^{4}$ structure solution program using charge flipping and refined with the ShelXL ${ }^{5}$ refinement package using least-squares minimization. All nonhydrogen atoms were refined with anisotropic displacement parameters. The hydrogen atoms on the ligands were placed in idealized positions and refined using a riding model. Guest molecules in complex $\mathbf{3}$ are significantly disordered and could not be modeled properly due to the lack of well define atomic positions, and PLATON ${ }^{6}$ and SQUEEZE software were employed to calculate the diffraction contribution of the solvent molecules and thereby produce a set of solvent-free diffraction intensities. SQUEEZE results of $\mathbf{3}$ is described below. Topological analysis of compound was performed by using ToposPro software. ${ }^{7}$ The detailed crystallographic data and structure refinement parameters were summarized in Table S3. Selected bond lengths and angles for 1-8 were collected in Table S4. Crystallographic data for the structural analyses has been deposited at the Cambridge Crystallographic Data Centre (CCDC reference numbers:1943715-1943722). 


\section{SQUEEZE results for complex 3 is as follows:}

\begin{tabular}{|c|c|c|c|c|c|}
\hline \multicolumn{6}{|c|}{ platon_squeeze_void_nr } \\
\hline \multicolumn{6}{|c|}{ _platon_squeeze_void_average_x } \\
\hline \multicolumn{6}{|c|}{ _platon_squeeze_void_average_y } \\
\hline \multicolumn{6}{|c|}{ platon_squeeze_void_average_z } \\
\hline \multicolumn{6}{|c|}{ _platon_squeeze_void_volume } \\
\hline \multicolumn{6}{|c|}{ platon_squeeze_void_count_electrons } \\
\hline \multicolumn{6}{|c|}{ platon_squeeze_void_content } \\
\hline 1 & 0.000 & 0.000 & -0.014 & 488 & $189^{\prime}$ \\
\hline 2 & 0.000 & 0.500 & 0.500 & 35 & $1^{\prime \prime}$ \\
\hline 3 & 0.167 & 0.833 & 0.833 & 35 & $1^{\prime \prime}$ \\
\hline 4 & 0.167 & 0.333 & 0.833 & 35 & $1^{\prime \prime}$ \\
\hline 5 & 0.333 & 0.667 & 0.160 & 493 & $191^{\prime \prime}$ \\
\hline 6 & 0.333 & 0.167 & 0.167 & 35 & $1^{\prime \prime}$ \\
\hline 7 & 0.500 & 0.500 & 0.500 & 35 & $1^{\prime \prime}$ \\
\hline 8 & 0.500 & 0.000 & 0.500 & 35 & $1^{\prime \prime}$ \\
\hline 9 & 0.667 & 0.333 & 0.826 & 493 & $191^{\prime \prime}$ \\
\hline 10 & 0.667 & 0.833 & 0.833 & 35 & $1^{\prime \prime}$ \\
\hline 11 & 0.833 & 0.167 & 0.167 & 35 & $1^{\prime \prime}$ \\
\hline 12 & 0.833 & 0.667 & 0.167 & 35 & $1^{\prime \prime}$ \\
\hline
\end{tabular}

That is, SQUEEZE gives 580 electrons/unit cell for the voids. If these electrons are all from $\mathrm{H}_{2} \mathrm{O}\left(10 \mathrm{e}^{-}\right.$), each unit cell has 58 (ca. 580/10) $\mathrm{H}_{2} \mathrm{O}$ molecules, and each formula unit has about three $\mathrm{H}_{2} \mathrm{O}$ molecules (since $\mathrm{Z}=18$ ).

\section{REFERENCES}

(1) Pang, X. H; Liu, J. F; Wei, G.; Shi, D. W; Bian, H.D; Liu, H. F; Yao, D.; Li, H. Y; Huang, F. P. Tracking the Formation of a Series of $\mathrm{Co}_{n}(\mathrm{n}=2,6,8)$ Clusters from Linear Co3 Precursor Clusters by Optimizing the Reaction Conditions. ChemistrySelect. 2018, 3, 7830-7835.

(2) Meshkova, S. B.; Kiriyak,A. V.; Gusev, A. N. Nischimenko, G. A. Shul'gin, V. F. IR luminescence of mixed-ligand complexes of $\mathrm{Nd}(\mathrm{III}), \mathrm{Yb}(\mathrm{III})$, and $\mathrm{Er}(\mathrm{III})$ with dibenzoylmethane and 1,2,4-triazoles. J. Appl. Spectrosc. 2012, 79, 708713.

(3) Dolomanov, O. V.; Bourhis, L. J.; Gildea, R. J.; Howard, J. A. K.; Puschmann, H. OLEX 2 : a complete structure solution, refinement and analysis program. J. Appl. Crystallogr. 2009, 42, 339-341.

(4) Sheldrick, G. M. A short history of SHELX. Acta. Crystallogr. A. 2008, A64, 112-122

(5) Sheldrick, G. M. Crystal structure refinement with SHELXL. Acta. Crystallogr. C. 2015, C71, 3-8.

(6) Spek, A. L. PLATON SQUEEZE: a tool for the calculation of the disordered solvent contribution to the calculated structure factors. Acta. Crystallogr. C., 2015, C71, 9-18.

(7) Blatov, V. A. IUCr CompComm Newsletter. 2006, 7, 4-38; https://topospro.com/. 
2. TGA curves of the complexes
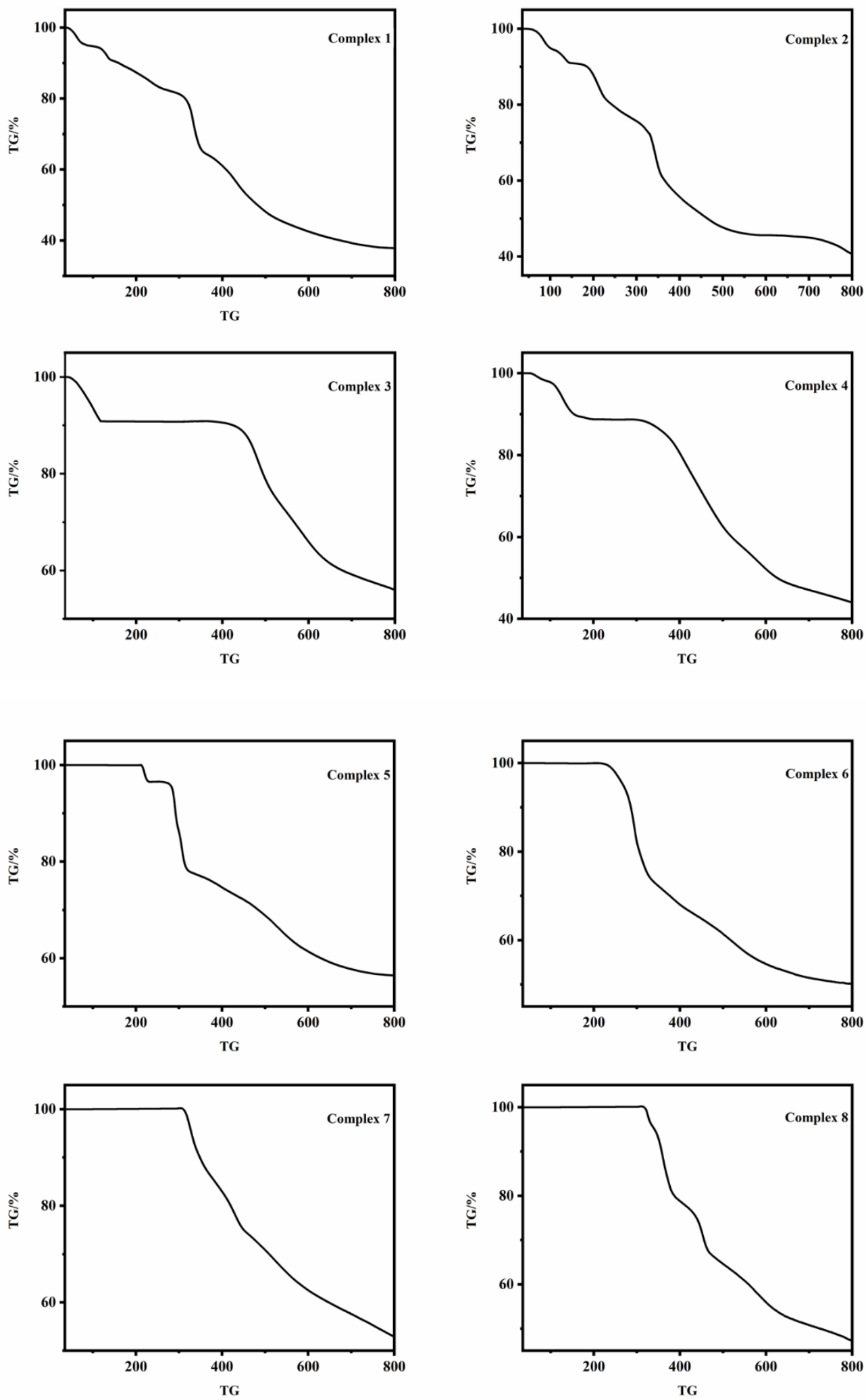

Figure S1. The TGA curves for the complexes 1-8. 
Thermogravimetric Analyses (TGA). Their thermogravimetric analyses are presented in Figures S1. Upon increasing the temperature, complexes $\mathbf{1}$ and $\mathbf{2}$ underwent a rapid continuous loss of weight, with a subsequent much slower weight loss until it decomposition. The weight loss of $35 \%$ and $30 \%$ before 340 and $330{ }^{\circ} \mathrm{C}$, respectively, which agrees well with free molecules and small molecular ligands $\left(\mathrm{H}_{2} \mathrm{O}, \mathrm{NO}_{3}^{-}\right.$; $\left.\mathrm{Ac}^{-}\right)$per formula unit (calcd: $35.60 \%$ for $1 ; 28.84 \%$ for 2 ). Complexes 3 and 4 underwent a rapid lost weight before $118^{\circ} \mathrm{C}$ and $188^{\circ} \mathrm{C}$, respectively. The $9.2 \%$ weight loss of $\mathbf{3}$ and $11.1 \%$ weight loss of $\mathbf{4}$ are corresponding to the removal of guest water molecules in $\mathbf{3}$ and coordinated water molecules in $\mathbf{4}$, respectively (calcd: $10.9 \%$ for $\mathbf{3} ; 12 \%$ for $\mathbf{4}$ ). No weight loss was found for the sample of complex $\mathbf{5}$ before $210^{\circ} \mathrm{C}$. Upon heating, a fast weight loss before $230^{\circ} \mathrm{C}$, then stabilized to $270{ }^{\circ} \mathrm{C}$, and then began to decompose. The weight loss of $3.2 \%$ before $230^{\circ} \mathrm{C}$ is due to the loss of the coordinated water molecules (calcd $2.76 \%$ ) per formula unit. For complexes 6,7 and 8 , they are stable at $225^{\circ} \mathrm{C}, 305^{\circ} \mathrm{C}$ and $315^{\circ} \mathrm{C}$ respectively, and then began to decompose.

\section{Structures of complexes}

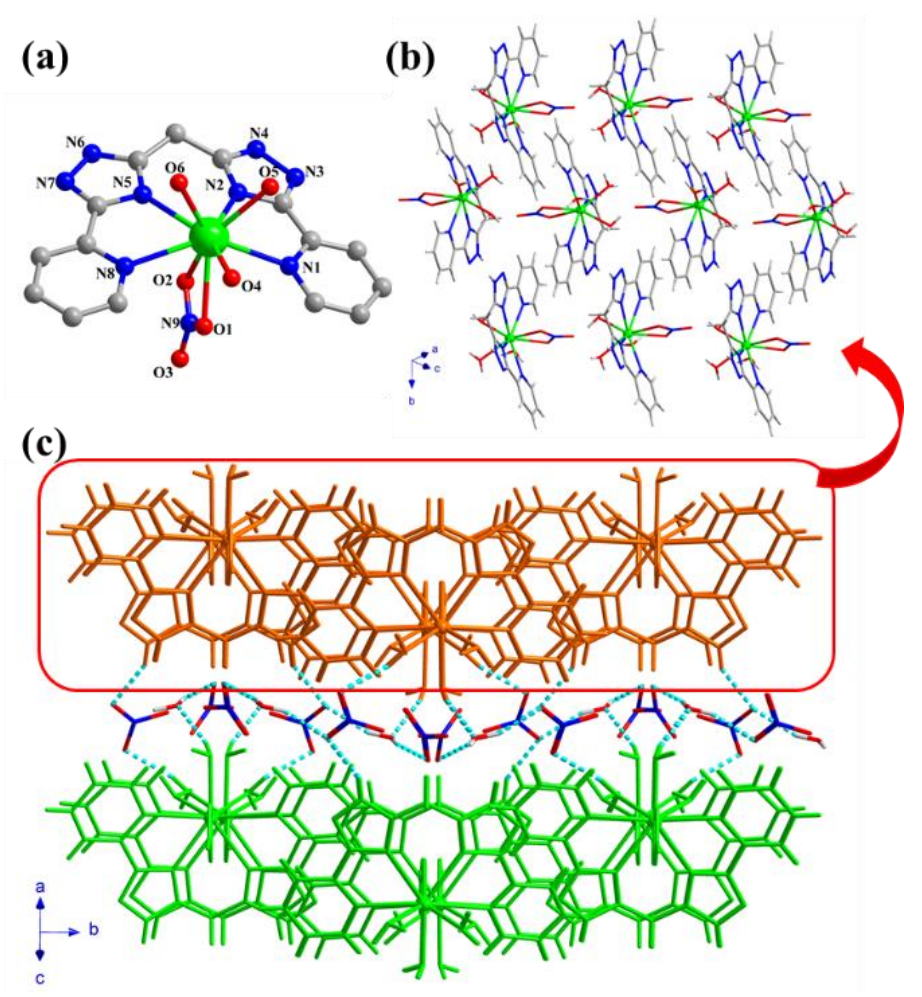

Figure S2. Coordination environment (a), two-dimensional layer (b) and packing arrangement (c) of 1. 
(a)

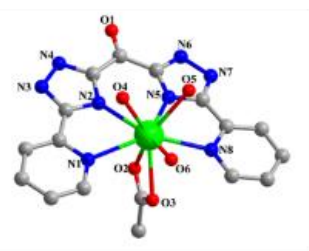

(c)
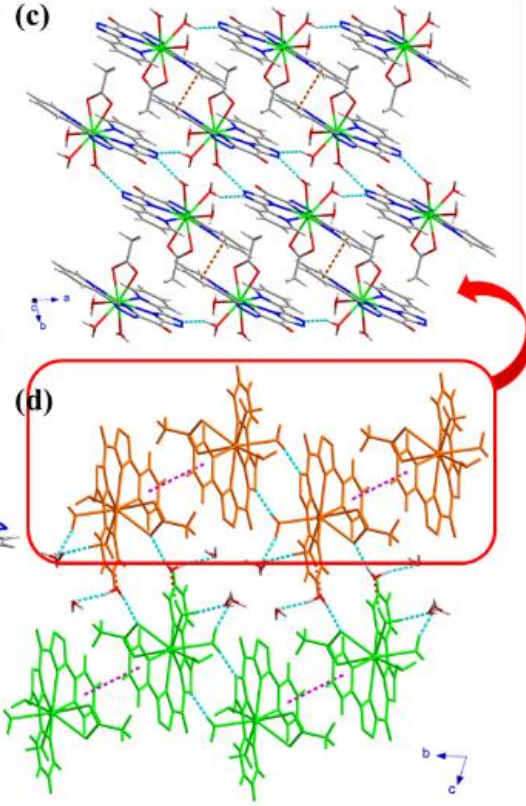

Figure S3. Coordination environment (a), two-dimensional layer (b), $\pi-\pi$ interaction (c) and packing arrangement (d) of $\mathbf{2}$.

(a)

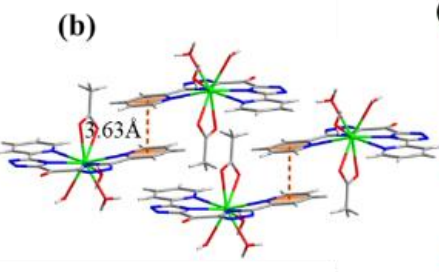

(c)

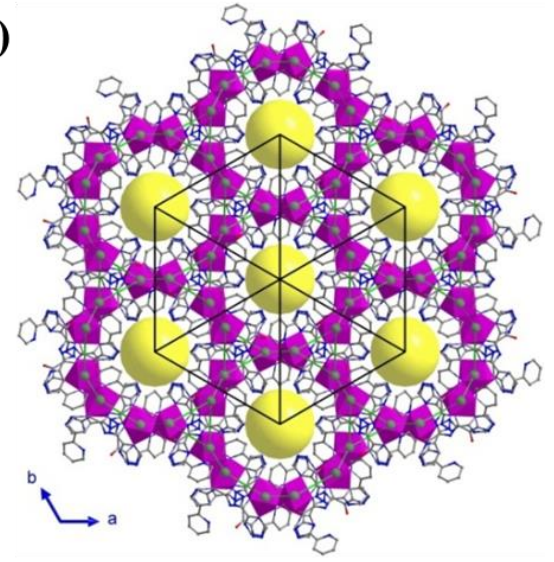

(d)

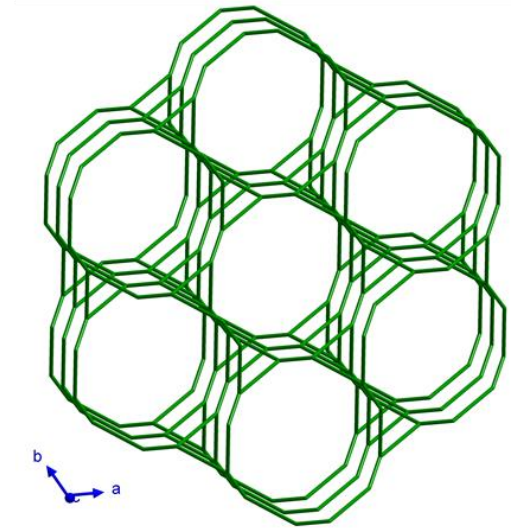

Figure S4. Coordination environment of $\mathbf{3}$ (a). The side view of 1D channel (b). 3D structure of $\mathbf{3}$ running along the c-axis (c). the topology of $\mathbf{3}(\mathrm{d})$ 
(a)

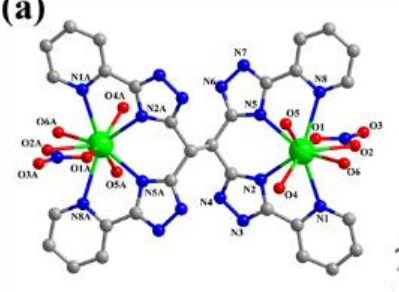

(b)
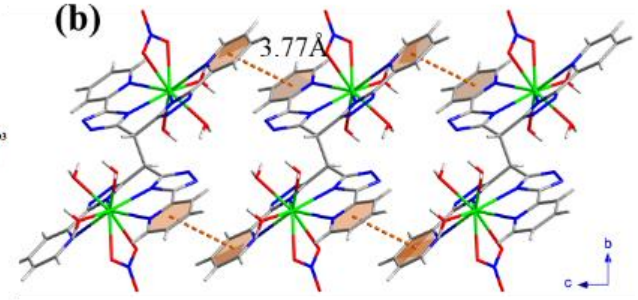

(c)

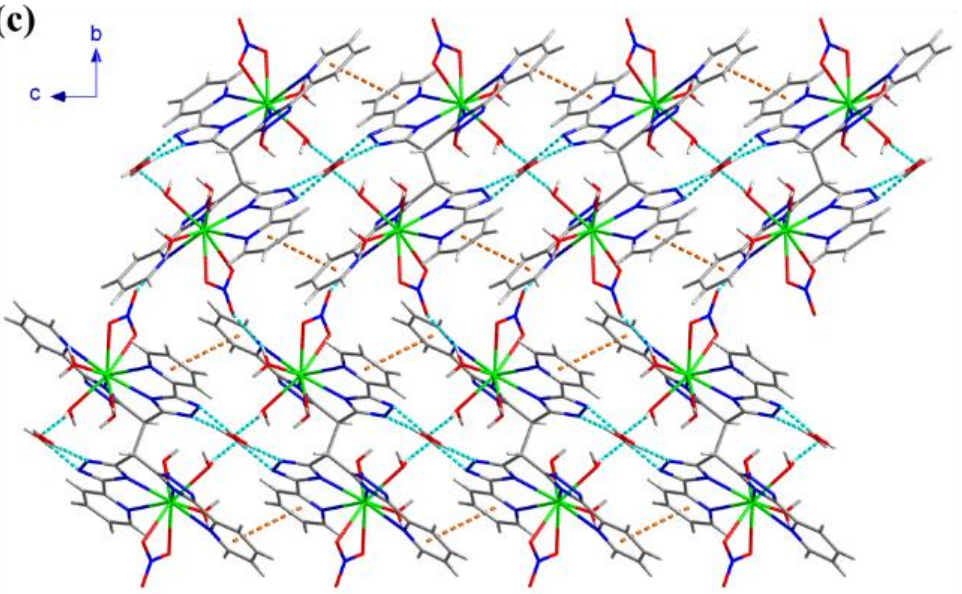

Figure S5. Coordination environment (a), $\pi-\pi$ interaction (b) and packing arrangement (c) of 4.

(a)

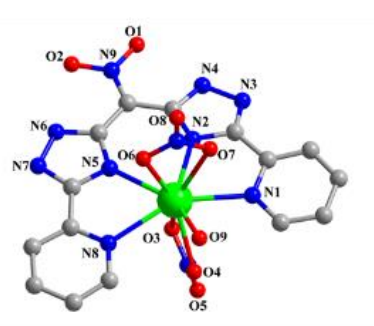

(c)

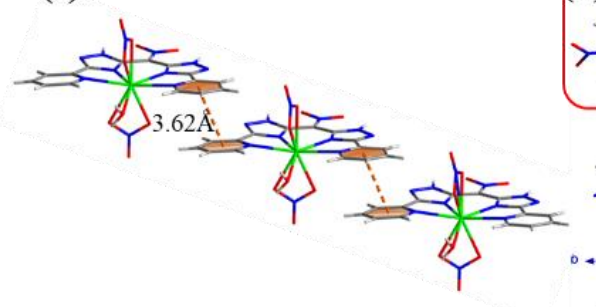

(b)

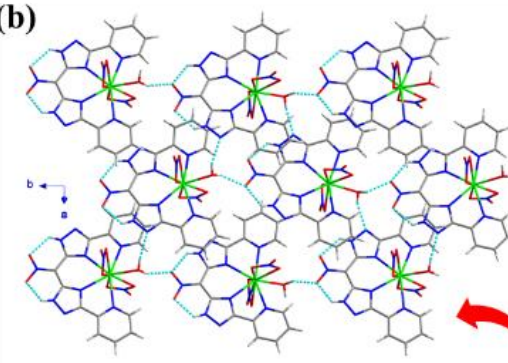

(d)

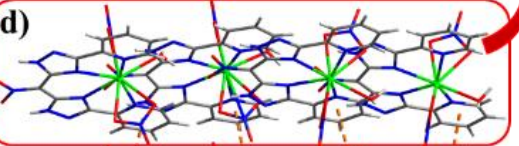

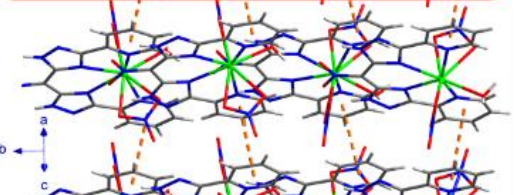

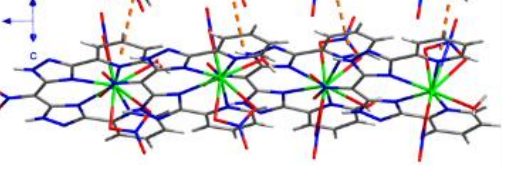

Figure S6. Coordination environment (a), two-dimensional layer (b), $\pi-\pi$ interaction (c) and packing arrangement (d) of complex $\mathbf{5}$. 

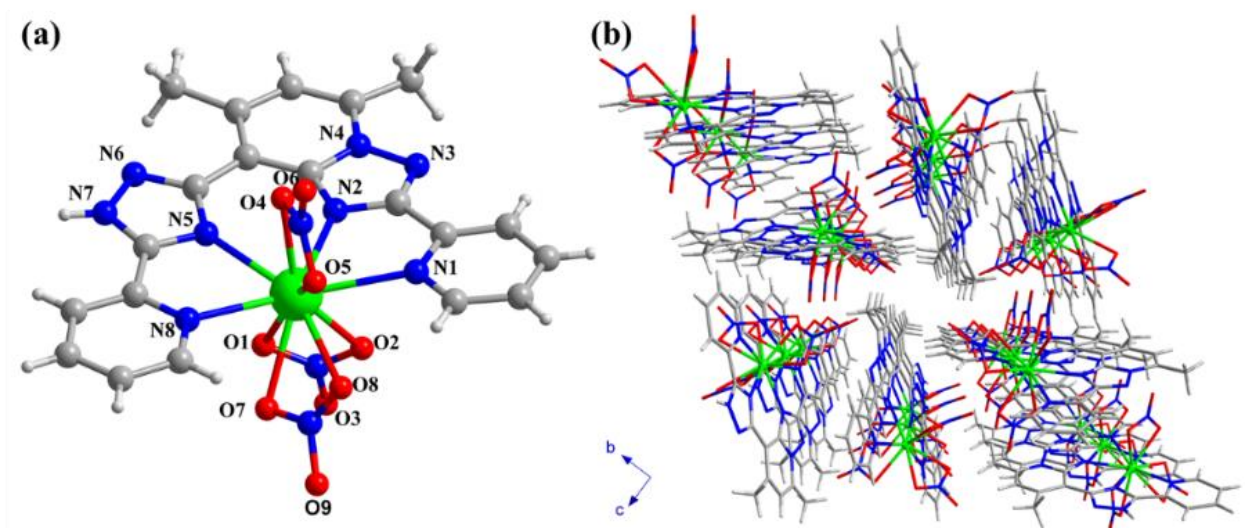

Figure S7. Coordination environment (a), and packing arrangement along $a$-axis (b) of 6 .

(a)

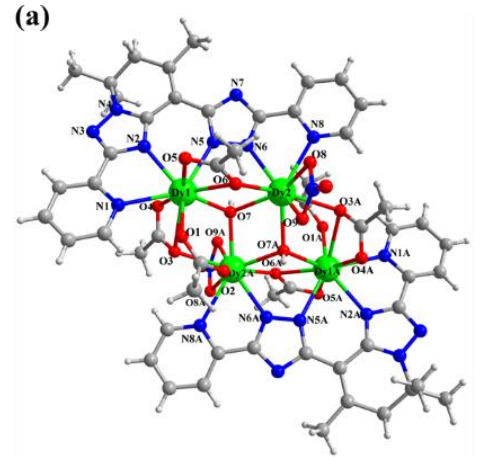

(b)

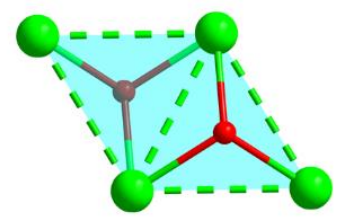

(c)

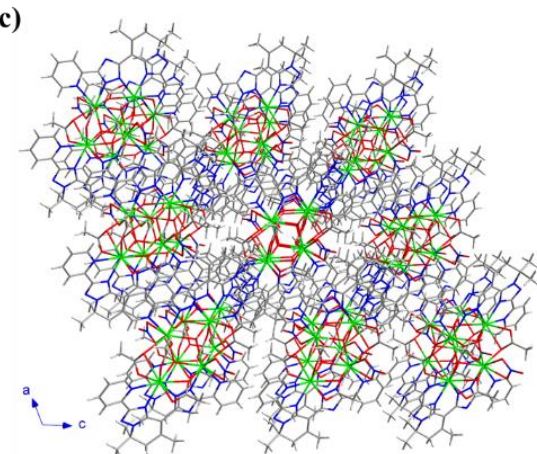

Figure S8. Perspective view of the tetranuclear core in 7 (a), The parallelogram core of Dy4 (b) and packing arrangement along $b$-axis. (c)

(a)

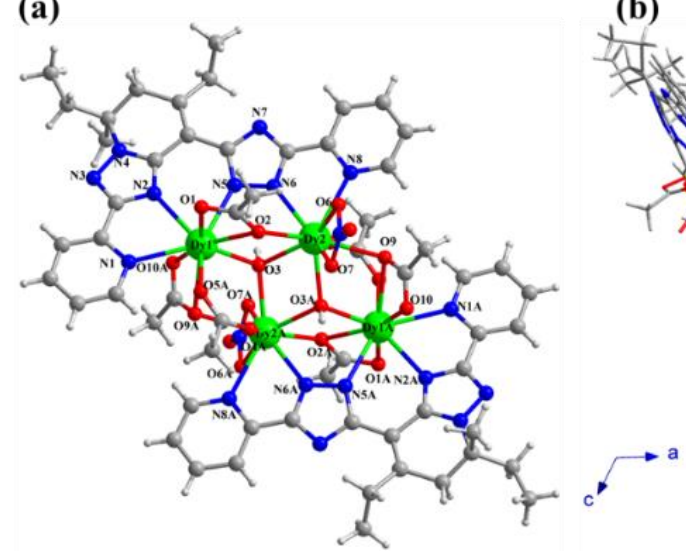

(b)

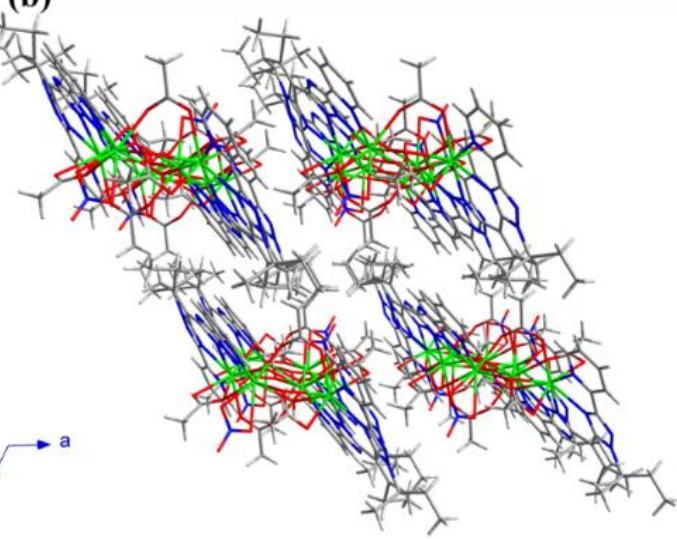

Figure S9. Perspective view of the tetranuclear core in $\mathbf{8}$ (a) and packing arrangement along $b$-axis. (b)

\section{SHAPE analysis of the Dy ${ }^{\text {III }}$ ions}

Table S1. SHAPE analysis of the Dy ${ }^{\text {III }}$ ions in complexes 1-8

\begin{tabular}{cccccc}
\hline complex & $\begin{array}{c}\text { Structure } \\
\text { [ML9] }\end{array}$ & $\begin{array}{c}\text { Capped square } \\
\text { antiprism J10 }\end{array}$ & $\begin{array}{c}\text { Spherical capped } \\
\text { square antiprism }\end{array}$ & $\begin{array}{c}\text { Tricapped trigonal } \\
\text { prism J51 }\end{array}$ & $\begin{array}{c}\text { Spherical tricapped } \\
\text { trigonal }\end{array}$ \\
$\mathbf{1}$ & Dy1 & 1.873 & $\mathbf{0 . 9 9 4}$ & 3.597 & 1.897 \\
\hline
\end{tabular}




\begin{tabular}{rccccc}
\hline $\mathbf{2}$ & Dy1 & 1.897 & $\mathbf{0 . 7 6 4}$ & 3.378 & 1.667 \\
$\mathbf{4}$ & Dy1 & 2.082 & $\mathbf{1 . 0 1 9}$ & 3.333 & 1.571 \\
$\mathbf{5}$ & Dy1 & 2.896 & $\mathbf{1 . 6 7 0}$ & 3.582 & 2.143 \\
& Dy2 & 2.649 & $\mathbf{1 . 4 0 6}$ & 4.136 & 2.586 \\
$\mathbf{7}$ & Dy1 & 3.597 & $\mathbf{2 . 4 1 6}$ & 3.228 & 3.265 \\
& Dy2 & 2.323 & $\mathbf{1 . 6 0 7}$ & 3.030 & 2.296 \\
$\mathbf{8}$ & Dy1 & 3.449 & $\mathbf{2 . 2 8 8}$ & 3.199 & 3.149 \\
& Dy2 & 2.471 & $\mathbf{1 . 7 3 8}$ & 3.145 & 2.362 \\
& {$[\mathbf{M L 8}]$} & Square antiprism & Triangular & Biaugmented trigonal & Biaugmented \\
& & & dodecahedron & prism J50 & trigonal prism \\
$\mathbf{3}$ & Dy1 & 3.292 & $\mathbf{1 . 3 4 1}$ & 3.445 & 2.697 \\
& [ML10] & Bicapped square & Sphenocorona & Staggered & Tetradecahedron \\
& & antiprism (J17) & $($ J87) & Dodecahedron (2:6:2) & $(2: 6: 2)$ \\
& Dy1 & 6.830 & $\mathbf{3 . 3 6 4}$ & 5.341 & 5.509 \\
\hline
\end{tabular}

\section{Ligand details}

Table S2. Ligand details

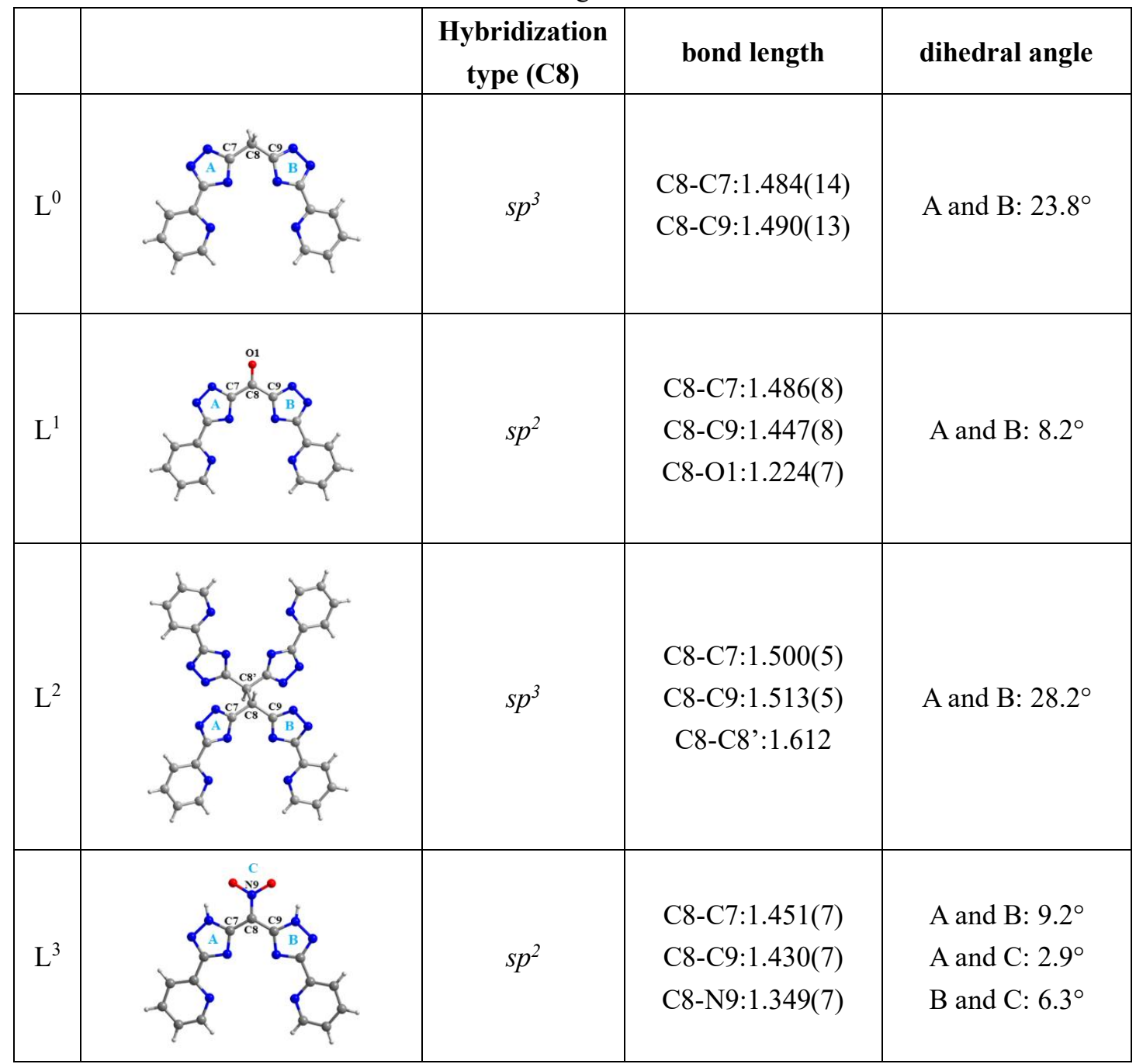




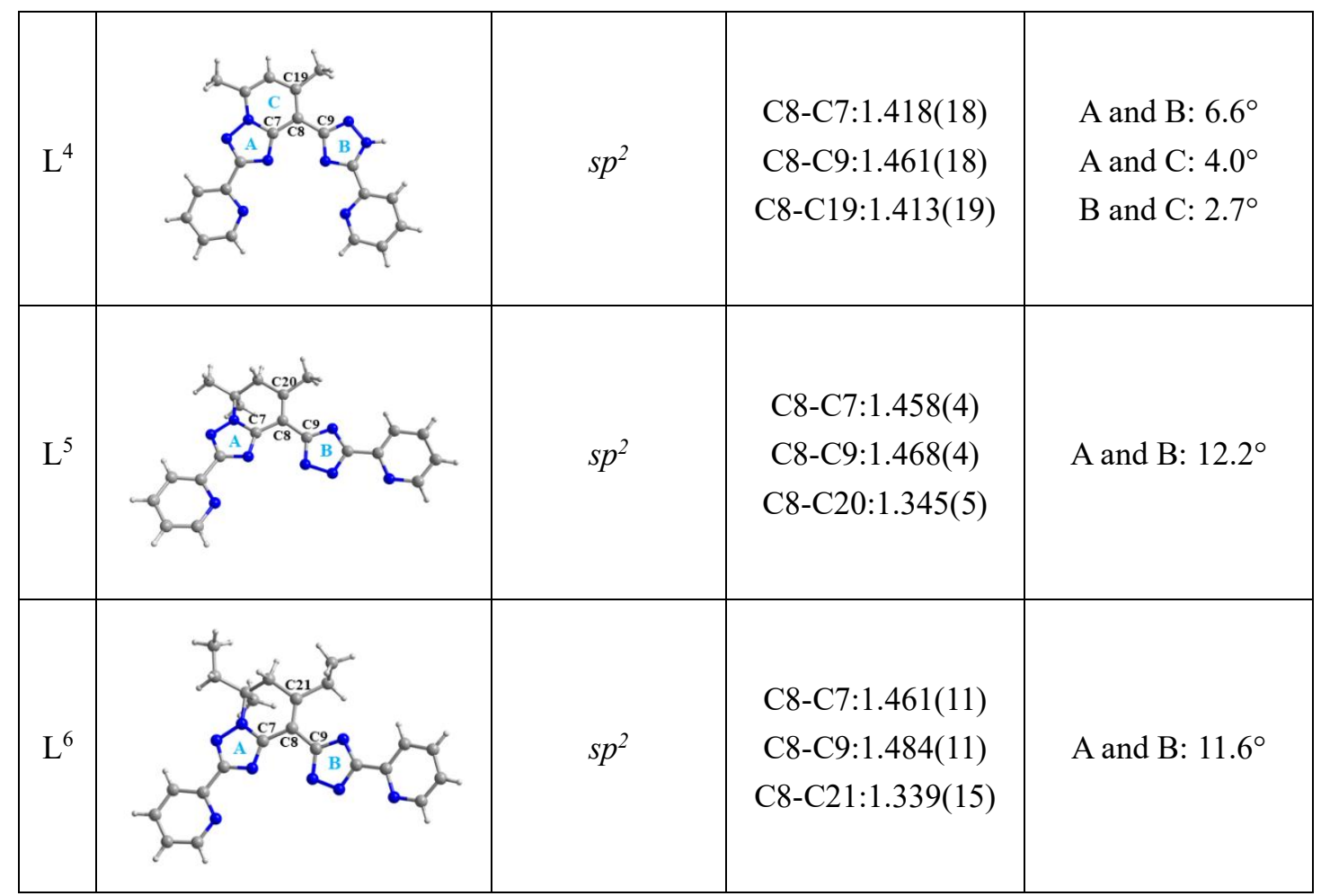

6. PXRD data of the complexes
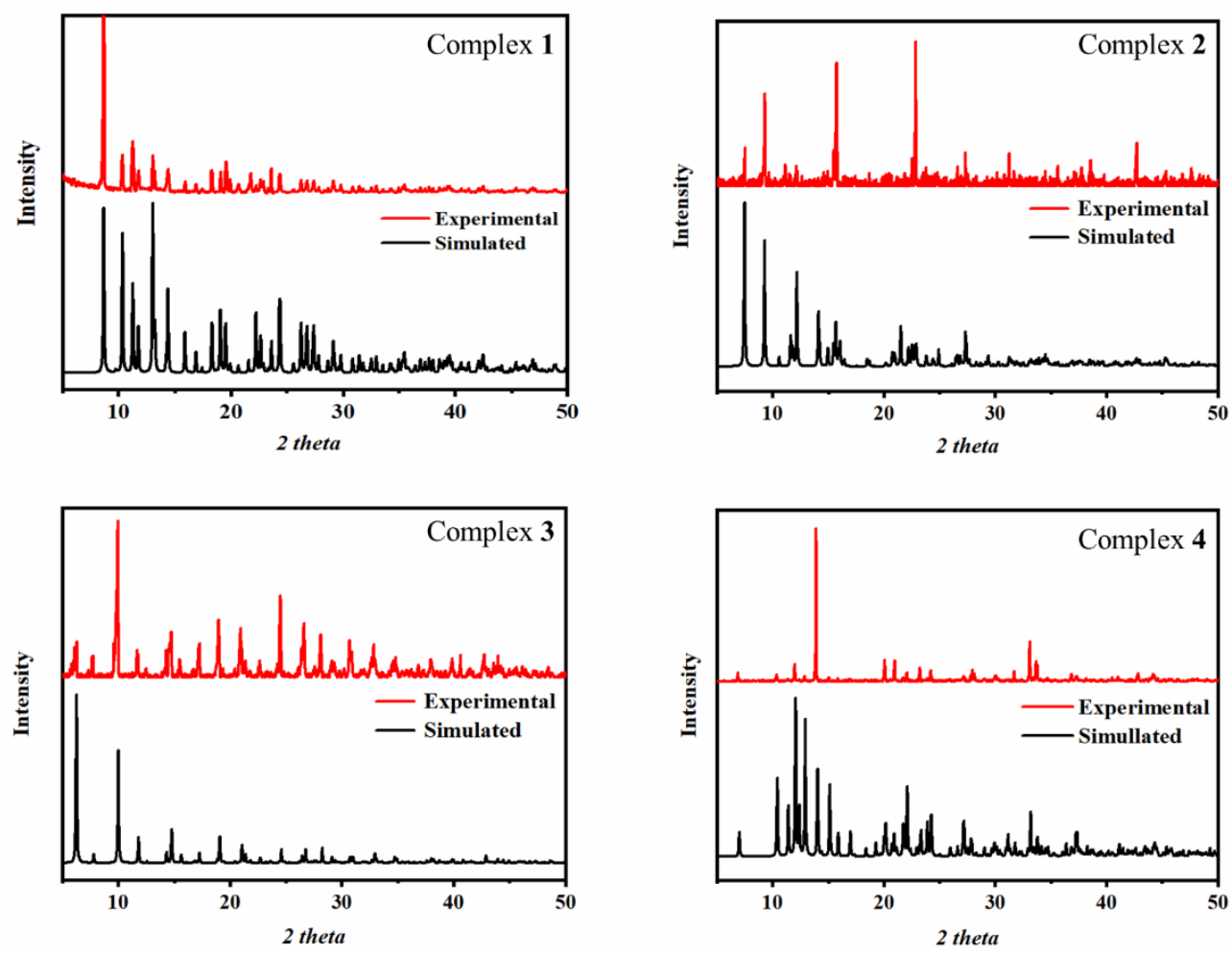

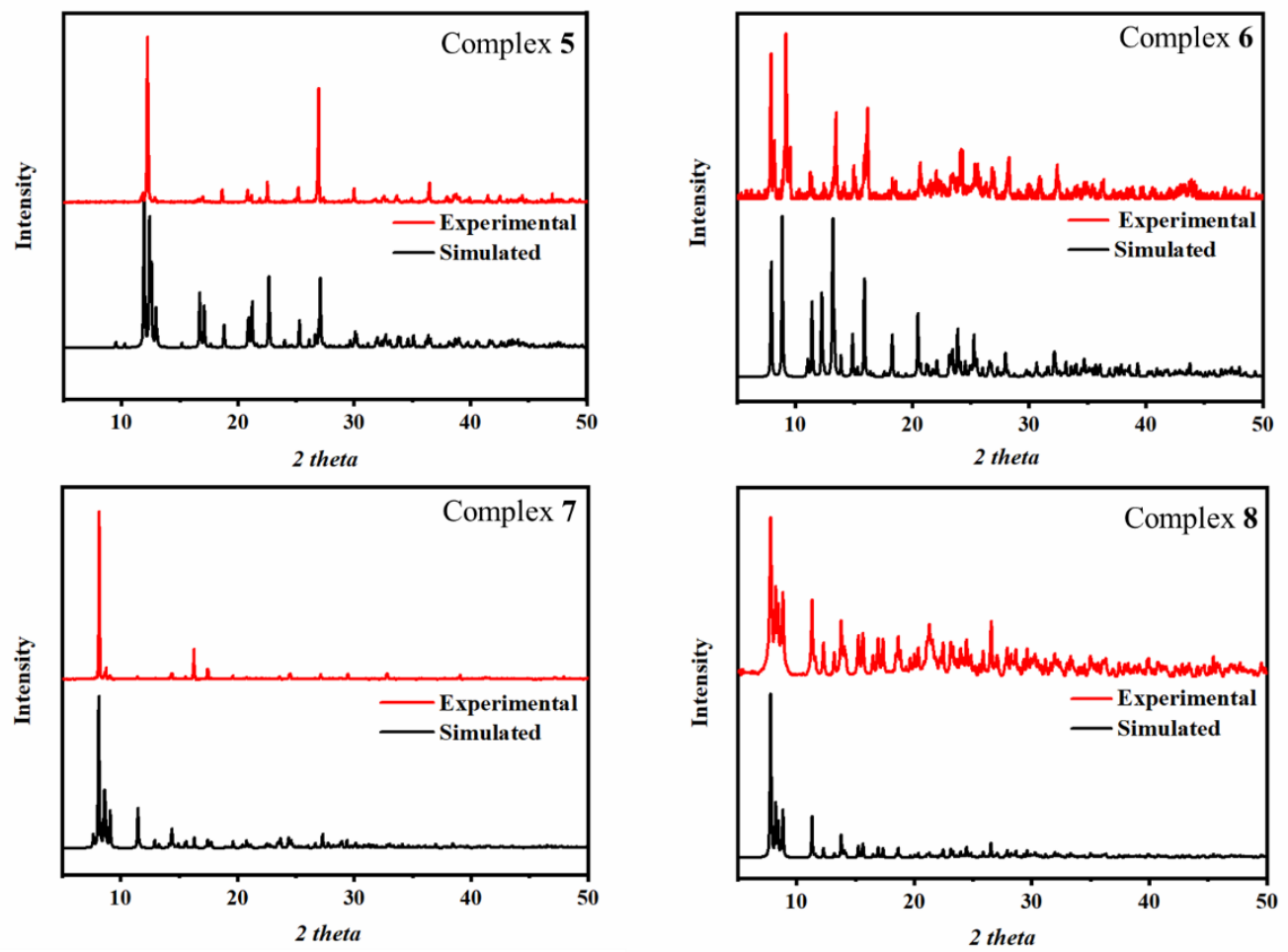

Figure S10. PXRD patterns and simulated for complexes 1-8.

\section{ESI-MS of the complexes}

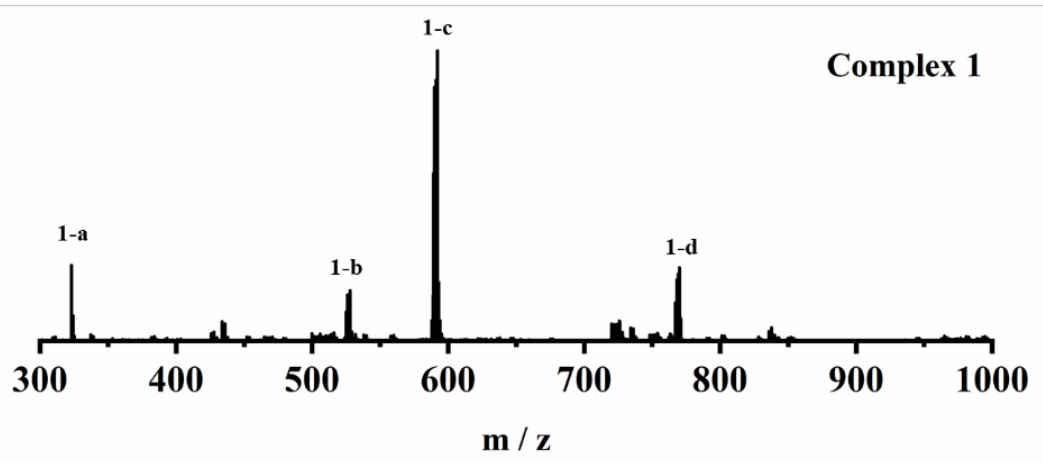

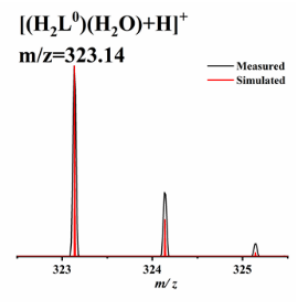

1-a

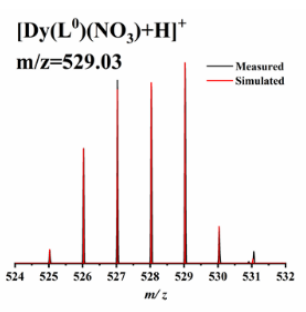

1-b
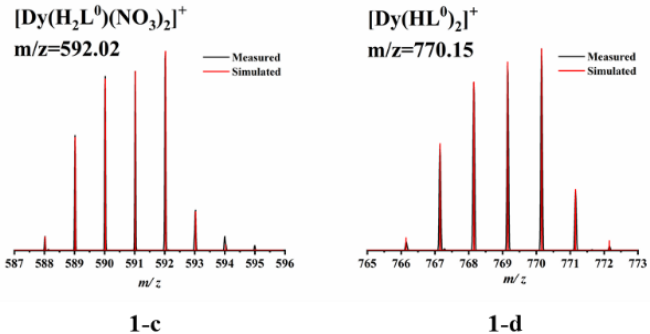

Figure S11. ESI-MS of complex 1. 

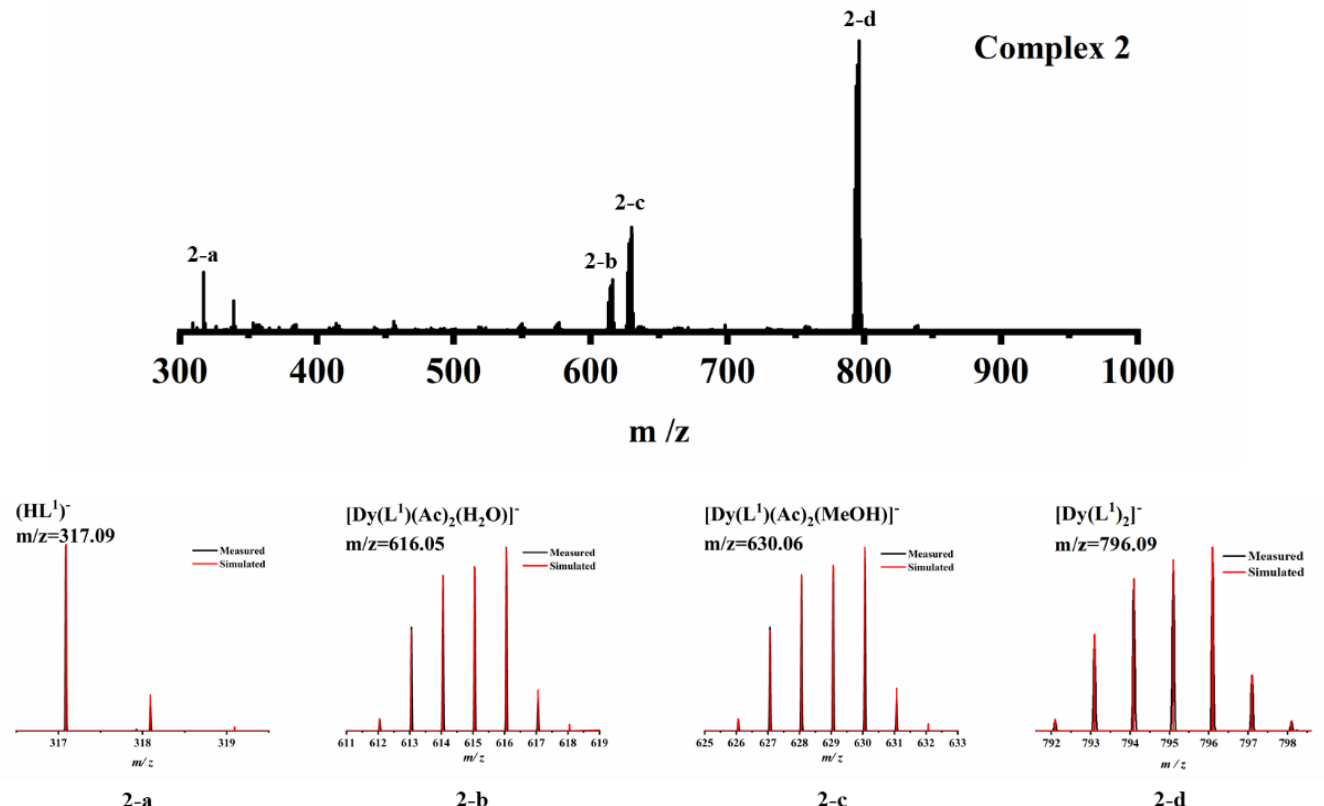

Figure S12. ESI-MS of complex 2.
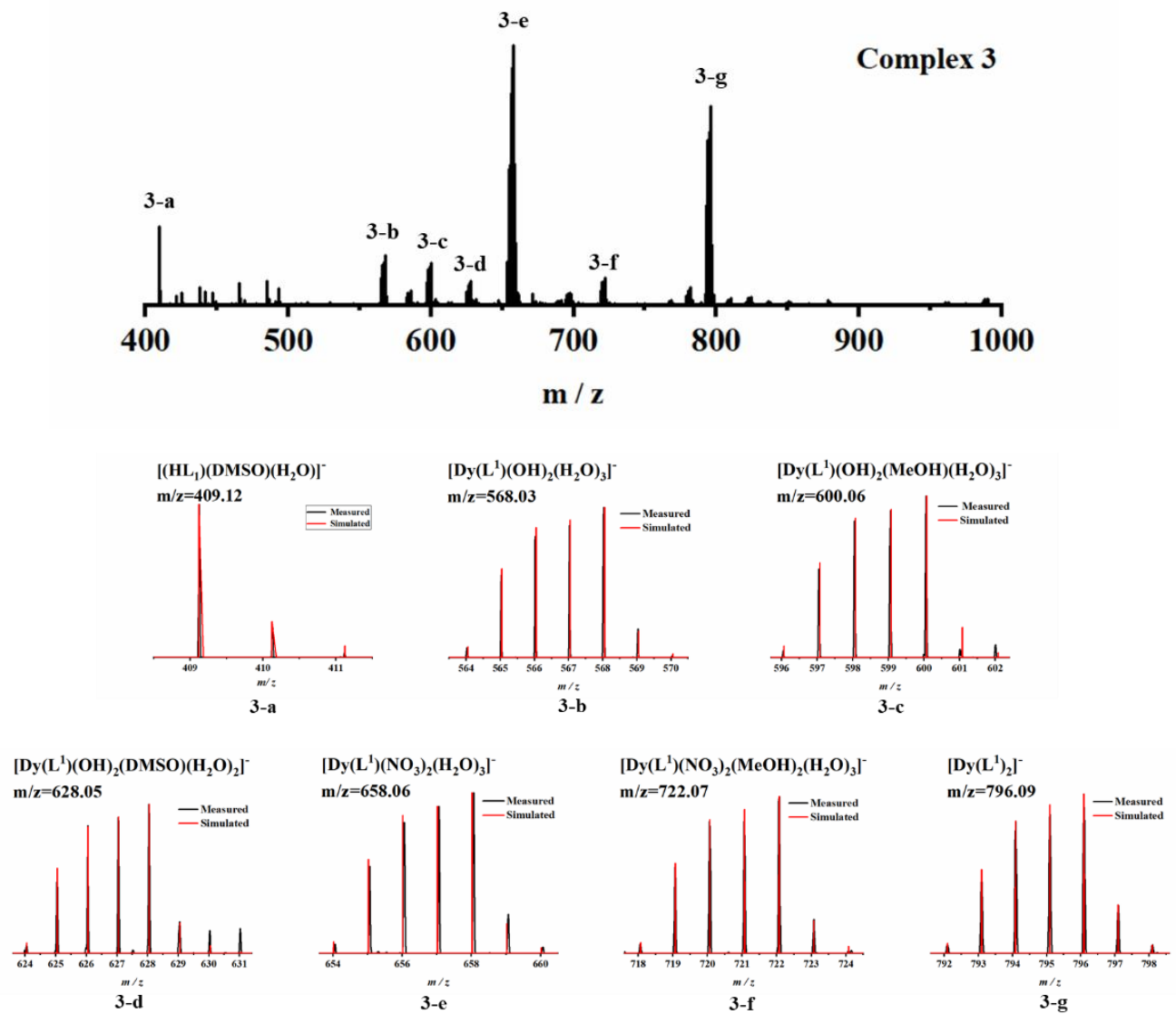

Figure S13. ESI-MS of complex 3. 

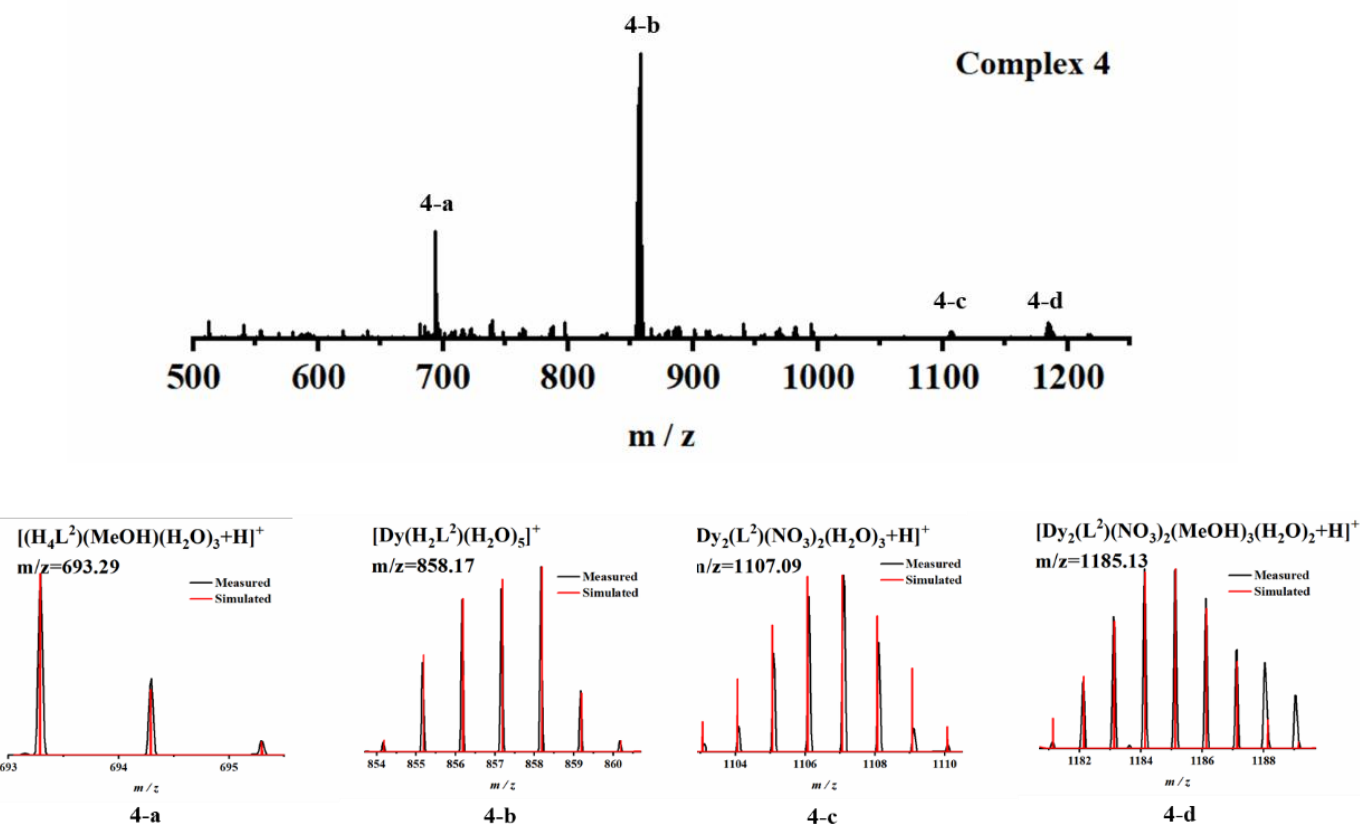

Figure S14. ESI-MS of complex 4.
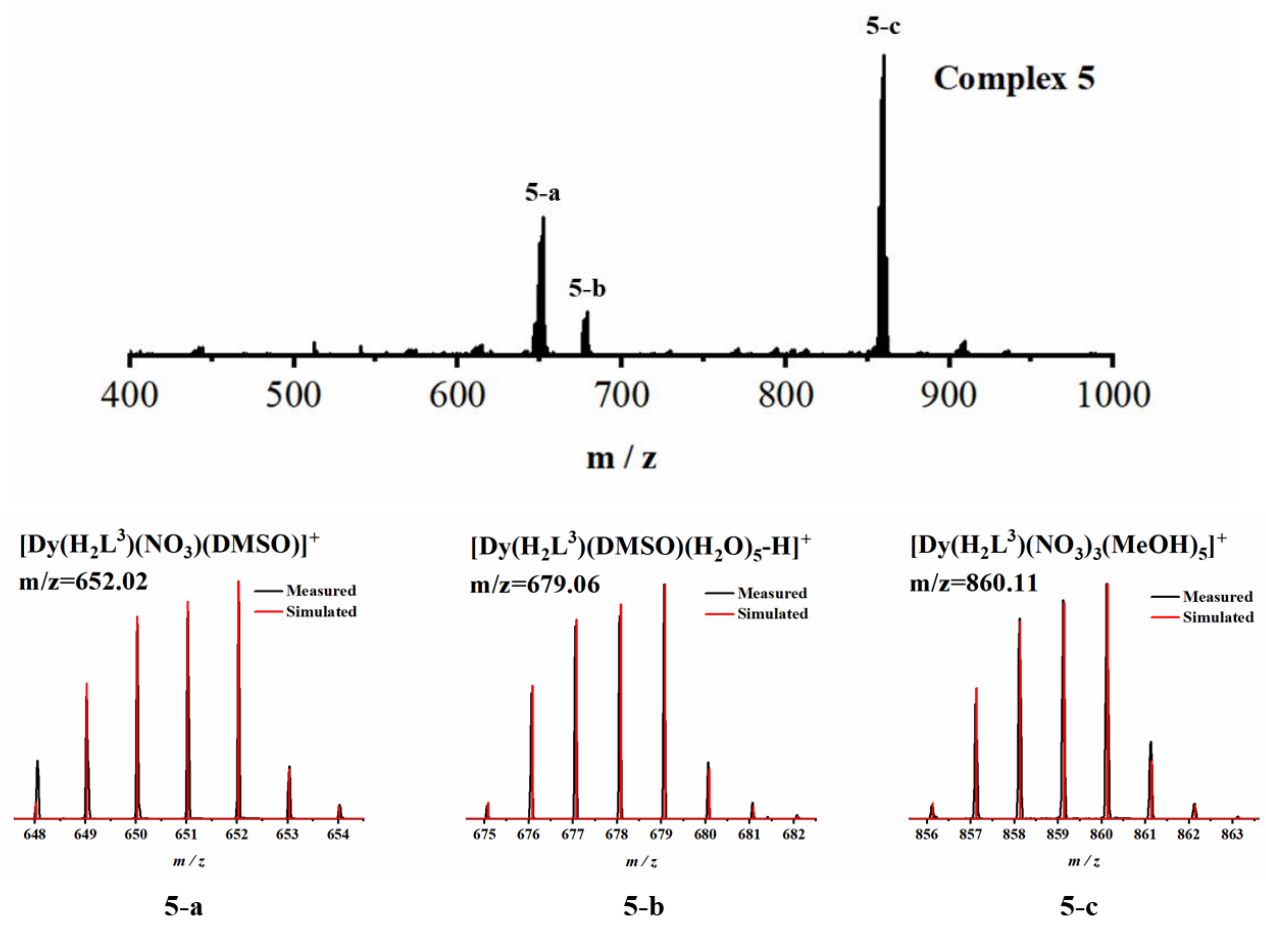

Figure S15. ESI-MS of complex 5. 

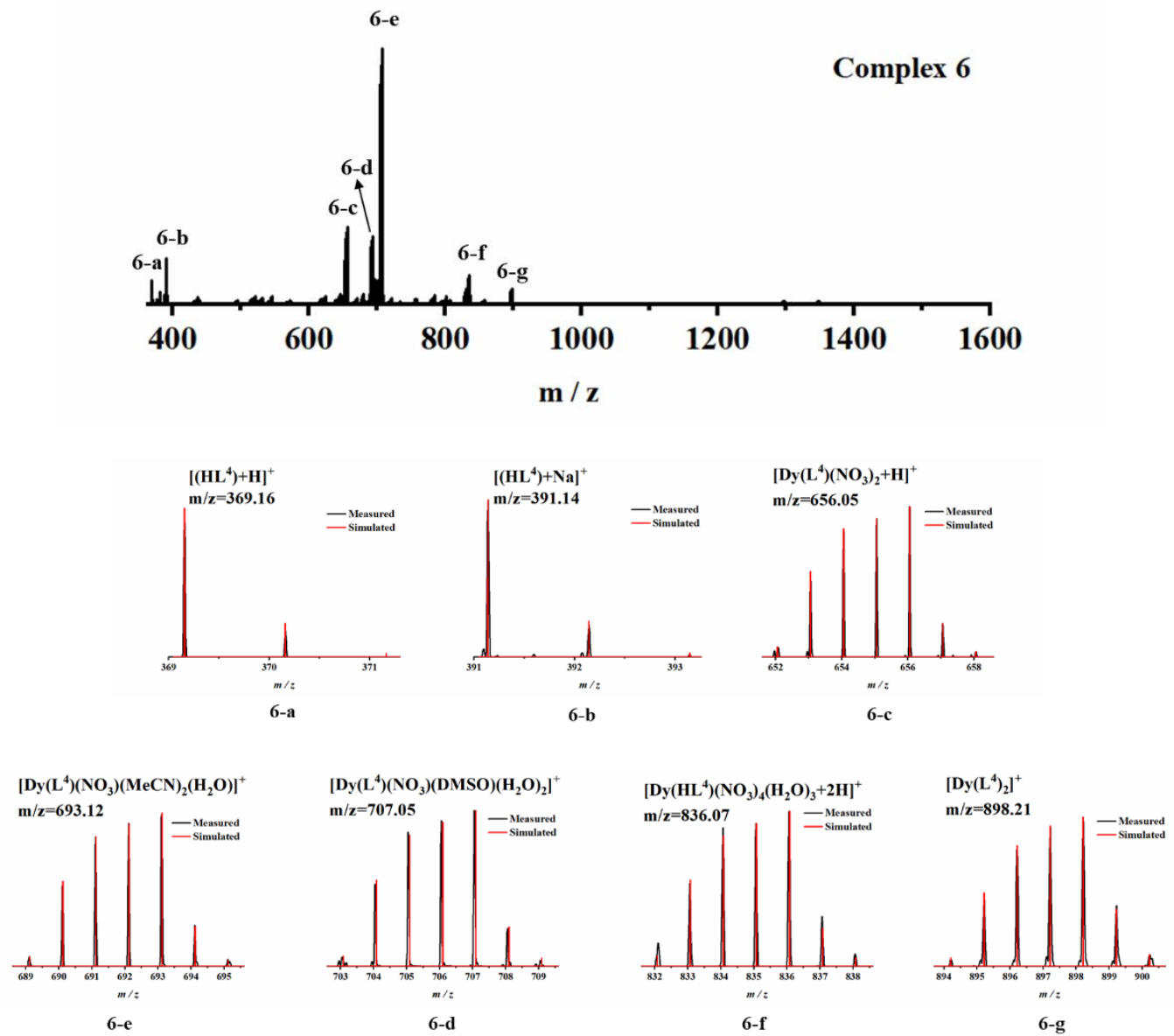

Figure S16. ESI-MS of complex 6.

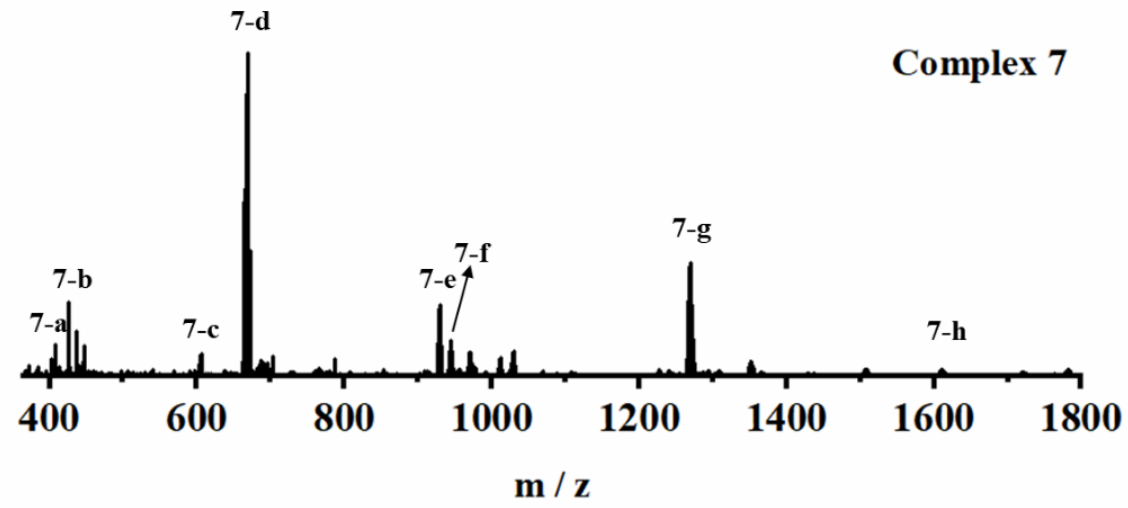



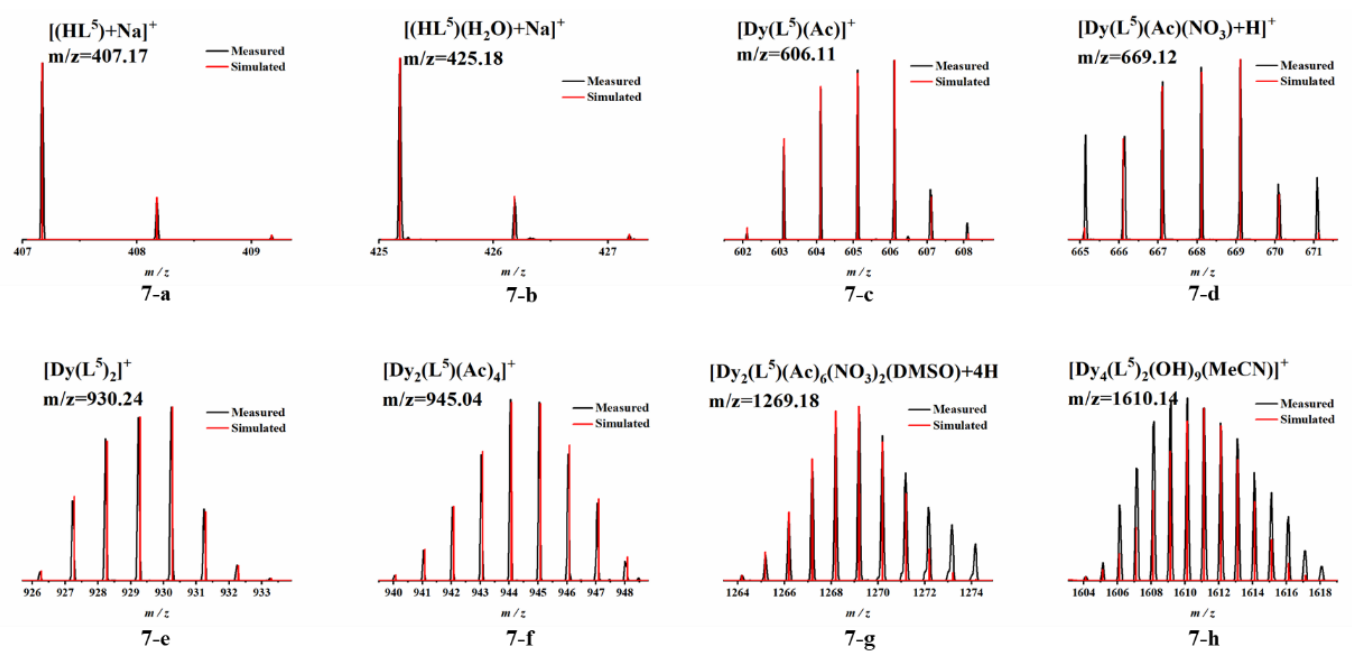

Figure S17. ESI-MS of complex 7.
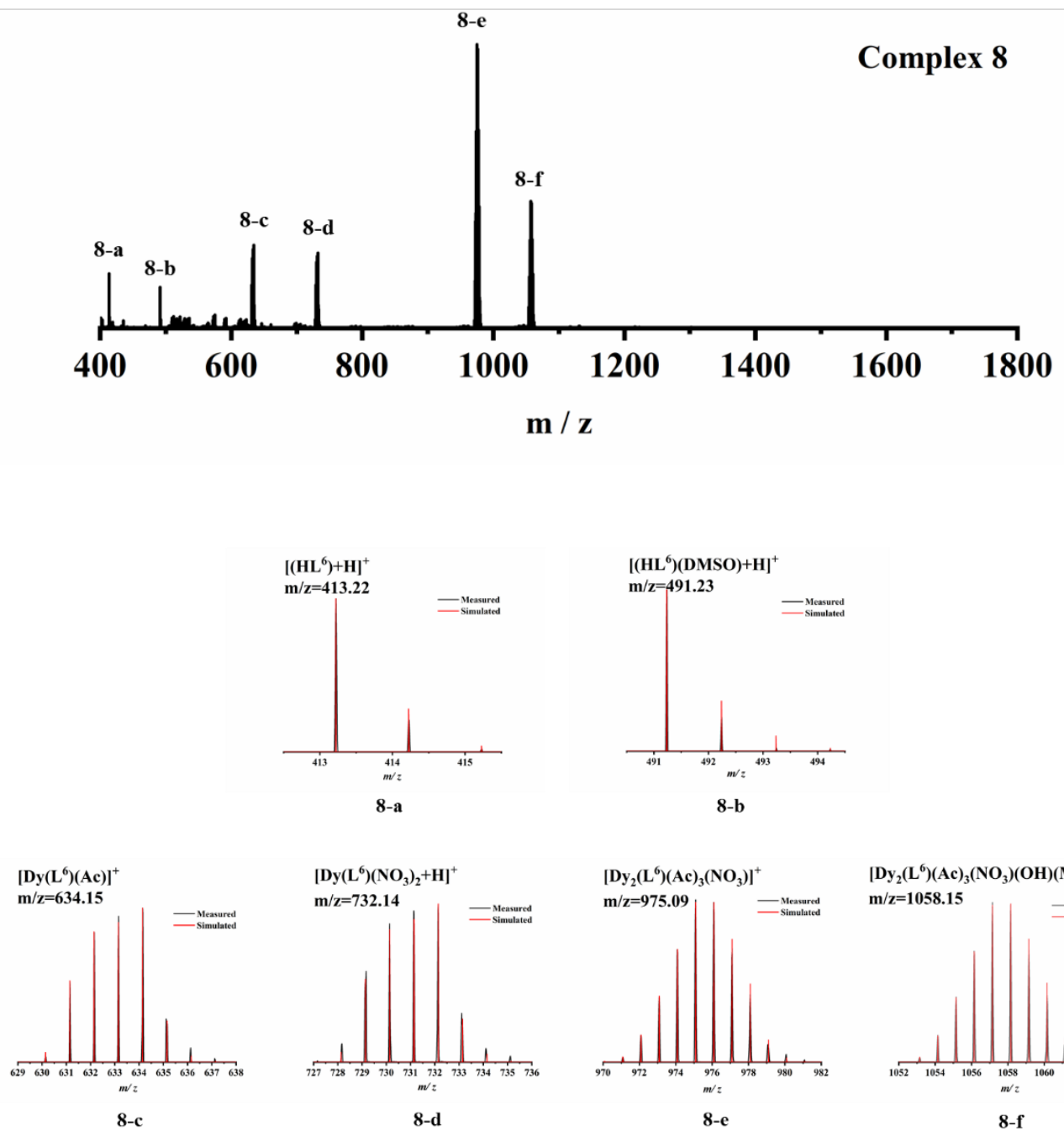

$\left[\mathrm{Dy}_{2}\left(\mathrm{~L}^{6}\right)(\mathrm{Ac})_{3}\left(\mathrm{NO}_{3}\right)(\mathrm{OH})(\mathrm{MeOH})_{2}+\mathrm{H}^{+}\right.$

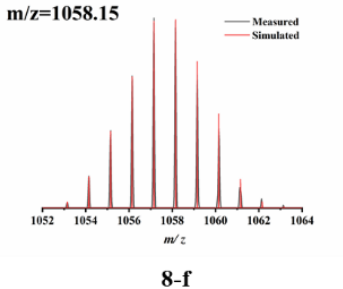

Figure S18. ESI-MS of complex 8. 


\section{Magnetism}
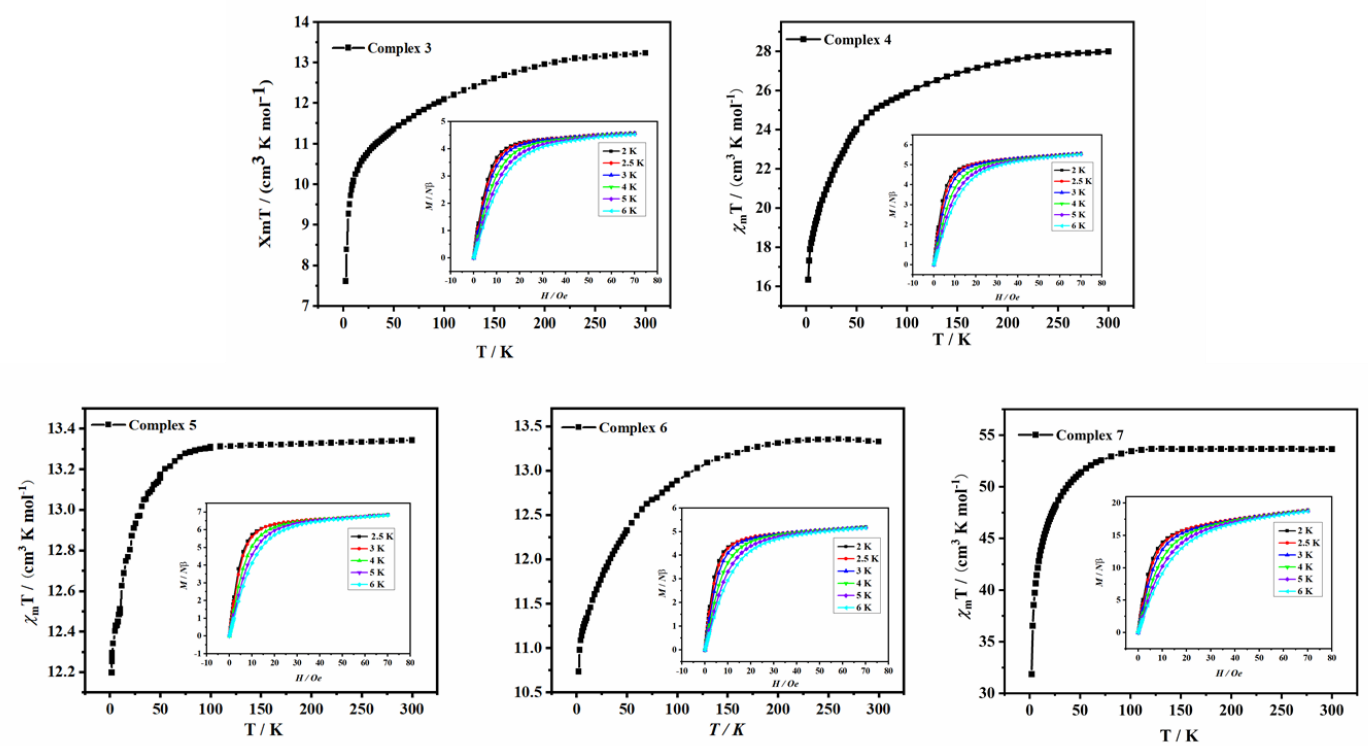

Figure S19. Plots of $\chi_{M} T$ vs $T$ at the applied field of $0.1 \mathrm{~T}$ of complex 3-7. Inset: Plots of M vs $\mathrm{H}$ for complexes 3-7 at the indicated temperatures.
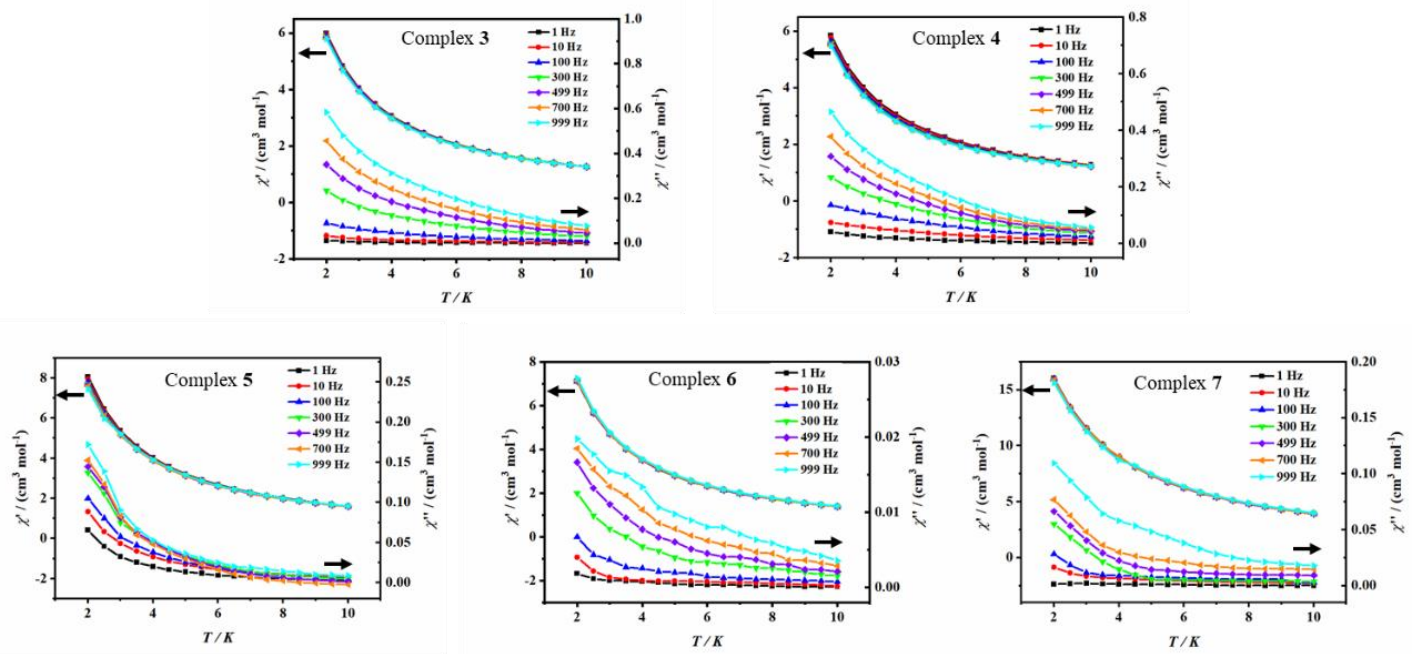

Figure S20. Data for complexes 3-7. Plot of $\chi^{\prime}$ and $\chi^{\prime \prime}$ versus temperature at different wave frequencies in the presence of a zero applied dc field.

\section{Crystallographic data and selected bond lengths $[\AA]]$ and angles $\left[{ }^{\circ}\right]$ for the complexes}

Table S3. Crystal Data and Refinement Parameters for complexes 1-8

\begin{tabular}{lllll}
\hline Identification code & $\mathbf{1}$ & $\mathbf{2}$ & $\mathbf{3}$ & $\mathbf{4}$ \\
\hline Empirical formula & $\mathrm{C}_{15} \mathrm{H}_{20} \mathrm{DyN}_{11} \mathrm{O}_{13}$ & $\mathrm{C}_{17} \mathrm{H}_{26} \mathrm{DyN}_{8} \mathrm{O}_{10.5}$ & $\mathrm{C} 15 \mathrm{H} 9 \mathrm{DyN} 8 \mathrm{O} 2$ & $\mathrm{C}_{30} \mathrm{H}_{34} \mathrm{Dy}_{2} \mathrm{~N}_{18} \mathrm{O}_{14}$ \\
Formula weight $(\mathrm{M})$ & 724.92 & 672.95 & 495.80 & 1195.75 \\
Crystal system & Monoclinic & Triclinic & Trigonal & Monoclinic \\
Space group & $P 2_{1} / \mathrm{n}$ & $P-1$ & $R-3$ & $P 2_{1} / \mathrm{n}$ \\
\hline
\end{tabular}




\begin{tabular}{|c|c|c|c|c|}
\hline $\mathrm{a}(\AA)$ & $11.6543(9)$ & $8.7529(3)$ & $28.2180(18)$ & $8.663(3)$ \\
\hline $\mathrm{b}(\AA)$ & $15.6814(11)$ & $12.0135(5)$ & $28.2180(18)$ & $25.211(10)$ \\
\hline c $(\AA)$ & $13.9918(17)$ & $12.6988(5)$ & $12.7757(8)$ & $9.590(4)$ \\
\hline$a\left(^{\circ}\right)$ & 90 & $72.047(4)$ & 90 & 90 \\
\hline$\beta\left(^{\circ}\right)$ & $107.375(10)$ & $74.734(3)$ & 90 & $110.028(6)$ \\
\hline$g\left({ }^{\circ}\right)$ & 90 & $77.234(3)$ & 120 & 90 \\
\hline $\mathrm{V} /\left(\AA^{3}\right)$ & $2440.4(4)$ & $1211.17(8)$ & $8809.8(13)$ & $1967.8(13)$ \\
\hline Z & 4 & 2 & 18 & 2 \\
\hline $\operatorname{Dc}\left(\mathrm{Mg} \mathrm{m}^{-3}\right)$ & 1.973 & 1.843 & 1.682 & 2.018 \\
\hline $\mathrm{F}(000)$ & 1428 & 666 & 4266 & 1168 \\
\hline Reflections collected & $13210 / 4460$ & $11645 / 4426$ & $32747 / 3589$ & $22010 / 3612$ \\
\hline unique & $\mathrm{R}($ int $)=0.0797$ & $\mathrm{R}($ int $)=0.0435$ & $\mathrm{R}($ int $)=0.0537$ & $\mathrm{R}($ int $)=0.0457$ \\
\hline Goodness-of-fit on F2 & 1.032 & 1.072 & 1.195 & 1.056 \\
\hline Final $\mathrm{R}$ indices & $\mathrm{R} 1=0.0594$ & $\mathrm{R} 1=0.0337$ & $\mathrm{R} 1=0.0776$ & $\mathrm{R} 1=0.0248$ \\
\hline $\mathrm{I}>2 \sigma(\mathrm{I})$ & $\omega \mathrm{R} 2=0.1156$ & $\omega \mathrm{R} 2=0.0598$ & $\omega \mathrm{R} 2=0.1564$ & $\omega \mathrm{R} 2=0.0538$ \\
\hline $\mathrm{R}$ indices & $\mathrm{R} 1=0.1106$ & $\mathrm{R} 1=0.0408$ & $\mathrm{R} 1=0.0821$ & $\mathrm{R} 1=0.0331$ \\
\hline (all data) & $\omega \mathrm{R} 2=0.1473$ & $\omega \mathrm{R} 2=0.0729$ & $\omega \mathrm{R} 2=0.1584$ & $\omega \mathrm{R} 2=0.0567$ \\
\hline Identification code & 5 & 6 & 7 & 8 \\
\hline Empirical formula & $\mathrm{C}_{15} \mathrm{H}_{12} \mathrm{DyN}_{11} \mathrm{O}_{9}$ & $\mathrm{C}_{20} \mathrm{H}_{16} \mathrm{DyN}_{11} \mathrm{O}_{9}$ & $\mathrm{C}_{54} \mathrm{H}_{58} \mathrm{Dy}_{4} \mathrm{~N}_{18} \mathrm{O}_{20}$ & $\mathrm{C}_{58} \mathrm{H}_{66} \mathrm{Dy}_{4} \mathrm{~N}_{18} \mathrm{O}_{20}$ \\
\hline Formula weight (M) & 652.86 & 716.94 & 1929.18 & 1985.29 \\
\hline Crystal system & Monoclinic & Monoclinic & Triclinic & Triclinic \\
\hline Space group & $P 2_{1}$ & $P 2_{1} / \mathrm{n}$ & $P-1$ & $P-1$ \\
\hline $\mathrm{a}(\AA)$ & 9.43355 (14) & $8.9052(13)$ & $11.7843(2)$ & $12.0533(16)$ \\
\hline $\mathrm{b}(\AA)$ & $21.1740(3)$ & $19.886(3)$ & $12.8173(3)$ & $13.1981(18)$ \\
\hline $\mathrm{c}(\AA)$ & $10.36562(17)$ & $13.4934(19)$ & 13.0499 (3) & $13.4988(18)$ \\
\hline$a\left(^{\circ}\right)$ & 90 & & $105.7672(19)$ & $107.452(2)$ \\
\hline$\beta\left(^{\circ}\right)$ & $92.5077(14)$ & $95.293(3)$ & 104.5295 (17) & $111.052(2)$ \\
\hline$g\left(^{\circ}\right)$ & 90 & & $111.957(2)$ & $105.468(2)$ \\
\hline $\mathrm{V} /\left(\AA^{3}\right)$ & $2068.51(5)$ & $2379.3(6)$ & $1616.33(6)$ & $1734.5(4)$ \\
\hline
\end{tabular}




\begin{tabular}{lllll}
\hline $\mathrm{Z}$ & 4 & 4 & 1 & 1 \\
$\mathrm{Dc}\left(\mathrm{Mg} \mathrm{m}^{-3}\right)$ & 2.096 & 2.001 & 1.982 & 1.901 \\
$\mathrm{~F}(000)$ & 1268 & 1404 & 932 & 964 \\
Reflections collected & $31521 / 8434$ & $26441 / 4353$ & $30803 / 5917$ & $19442 / 6317$ \\
unique & $\mathrm{R}(\mathrm{int})=0.0541$ & $\mathrm{R}(\mathrm{int})=0.0664$ & $\mathrm{R}(\mathrm{int})=0.0268$ & $\mathrm{R}(\mathrm{int})=0.0264$ \\
Goodness-of-fit on $\mathrm{F} 2$ & 1.002 & 1.179 & 1.123 & 1.042 \\
Final $\mathrm{R}$ indices & $\mathrm{R} 1=0.0282$ & $\mathrm{R} 1=0.0758$ & $\mathrm{R} 1=0.0180$ & $\mathrm{R} 1=0.0324$ \\
$\mathrm{I}>2 \sigma(\mathrm{I})$ & $\omega \mathrm{R} 2=0.0553$ & $\omega \mathrm{R} 2=0.1873$ & $\omega \mathrm{R} 2=0.0413$ & $\omega \mathrm{R} 2=0.0996$ \\
$\mathrm{R}$ indices & $\mathrm{R} 1=0.0326$ & $\mathrm{R} 1=0.0911$ & $\mathrm{R} 1=0.0232$ & $\mathrm{R} 1=0.0433$ \\
$($ all data $)$ & $\omega \mathrm{R} 2=0.0575$ & $\omega \mathrm{R} 2=0.1949$ & $\omega \mathrm{R} 2=0.0439$ & $\omega \mathrm{R} 2=0.1058$ \\
\hline
\end{tabular}

Table S4. Selected bond length and bond angle for complexes 1-8

1

Atomic Distances [̊̊]

Dy1-N1 $2.548(8)$

Dy1-O2

$2.448(7)$

Dy1-N2

$2.457(8)$

Dy1-O4

$2.316(7)$

Dy1-N5

$2.471(8)$

Dy1-O5

$2.393(7)$

Dy1-N8

$2.584(8)$

Dy1-O6

$2.420(6)$

Dy1-O1

$2.525(8)$

\section{Bond Angles $\left[{ }^{\circ}\right]$}

$\begin{array}{llll}\text { N1-Dy1-N8 } & 142.5(3) & \text { O4-Dy1-N5 } & 144.0(2) \\ \text { N2-Dy1-N1 } & 66.0(3) & \text { O4-Dy1-N8 } & 83.5(3) \\ \text { N2-Dy1-N5 } & 69.5(3) & \text { O4-Dy1-O2 } & 119.6(3) \\ \text { N2-Dy1-N8 } & 131.1(3) & \text { O4-Dy1-O5 } & 77.4(3) \\ \text { N2-Dy1-O1 } & 114.2(2) & \text { O4-Dy1-O6 } & 80.5(2) \\ \text { N5-Dy1-N1 } & 131.6(3) & \text { O5-Dy1-N1 } & 71.8(3) \\ \text { N5-Dy1-N8 } & 64.6(3) & \text { O5-Dy1-N2 } & 76.8(2) \\ \text { N5-Dy1-O1 } & 112.0(3) & \text { O5-Dy1-N5 } & 115.1(3)\end{array}$




\begin{tabular}{|c|c|c|c|}
\hline $\mathrm{O} 1-\mathrm{Dy} 1-\mathrm{N} 1$ & $71.9(3)$ & O5-Dy1-N8 & $138.3(2)$ \\
\hline $\mathrm{O} 1-\mathrm{Dy} 1-\mathrm{N} 8$ & $70.6(3)$ & $\mathrm{O} 5-\mathrm{Dy} 1-\mathrm{O} 1$ & $132.3(2)$ \\
\hline $\mathrm{O} 2-\mathrm{Dy} 1-\mathrm{N} 1$ & $78.0(2)$ & $\mathrm{O} 5-\mathrm{Dy} 1-\mathrm{O} 2$ & $143.5(2)$ \\
\hline $\mathrm{O} 2-\mathrm{Dy} 1-\mathrm{N} 2$ & $72.4(3)$ & O5-Dy1-O6 & $67.5(2)$ \\
\hline $\mathrm{O} 2-\mathrm{Dy} 1-\mathrm{N} 5$ & $71.4(2)$ & O6-Dy1-N1 & $138.6(2)$ \\
\hline $\mathrm{O} 2-\mathrm{Dy} 1-\mathrm{N} 8$ & $78.0(2)$ & O6-Dy1-N2 & $110.3(3)$ \\
\hline $\mathrm{O} 2-\mathrm{Dy} 1-\mathrm{O} 1$ & $50.7(2)$ & O6-Dy1-N5 & $74.8(2)$ \\
\hline $\mathrm{O} 4-\mathrm{Dy} 1-\mathrm{N} 1$ & $83.9(3)$ & O6-Dy1-N8 & $73.0(3)$ \\
\hline $\mathrm{O} 4-\mathrm{Dy} 1-\mathrm{N} 2$ & $145.1(2)$ & O6-Dy1-O1 & $134.4(2)$ \\
\hline O4-Dy1-O1 & $68.9(3)$ & $\mathrm{O} 6-\mathrm{Dy} 1-\mathrm{O} 2$ & $142.4(2)$ \\
\hline \multicolumn{4}{|l|}{2} \\
\hline \multicolumn{4}{|c|}{ Atomic Distances $[\AA]]$} \\
\hline Dy $1-O 2$ & $2.402(3)$ & Dy $1-\mathrm{N} 1$ & $2.567(4)$ \\
\hline Dy1-O3 & $2.450(3)$ & Dy $1-\mathrm{N} 2$ & $2.444(4)$ \\
\hline Dy $1-O 4$ & $2.521(3)$ & Dy $1-\mathrm{N} 5$ & $2.453(4)$ \\
\hline Dy1-O5 & $2.442(4)$ & Dy $1-\mathrm{N} 8$ & $2.563(4)$ \\
\hline Dy1-O6 & $2.359(3)$ & & \\
\hline \multicolumn{4}{|l|}{ Bond Angles $\left[{ }^{\circ}\right]$} \\
\hline $\mathrm{O} 2-\mathrm{Dy} 1-\mathrm{O} 3$ & $53.49(11)$ & O5-Dy1-N8 & $73.29(13)$ \\
\hline $\mathrm{O} 2-\mathrm{Dy} 1-\mathrm{O} 4$ & $143.13(11)$ & $\mathrm{O} 6-\mathrm{Dy} 1-\mathrm{O} 2$ & $125.39(12)$ \\
\hline $\mathrm{O} 2-\mathrm{Dy} 1-\mathrm{O} 5$ & $144.31(12)$ & $\mathrm{O} 6-\mathrm{Dy} 1-\mathrm{O} 3$ & $71.93(12)$ \\
\hline $\mathrm{O} 2-\mathrm{Dy} 1-\mathrm{N} 1$ & $80.72(12)$ & O6-Dy1-O4 & $74.85(11)$ \\
\hline $\mathrm{O} 2-\mathrm{Dy} 1-\mathrm{N} 2$ & $76.41(12)$ & O6-Dy1-O5 & $74.25(12)$ \\
\hline $\mathrm{O} 2-\mathrm{Dy} 1-\mathrm{N} 5$ & $75.77(12)$ & O6-Dy1-N1 & $86.61(12)$ \\
\hline $\mathrm{O} 2-\mathrm{Dy} 1-\mathrm{N} 8$ & $79.70(12)$ & O6-Dy1-N2 & $142.81(12)$ \\
\hline O3-Dy1-O4 & $131.09(11)$ & O6-Dy1-N5 & $139.53(12)$ \\
\hline $\mathrm{O} 3-\mathrm{Dy} 1-\mathrm{N} 1$ & $73.90(12)$ & O6-Dy1-N8 & $82.99(11)$ \\
\hline O3-Dy1-N5 & $118.84(12)$ & $\mathrm{N} 2-\mathrm{Dy} 1-\mathrm{O} 3$ & $119.59(12)$ \\
\hline $\mathrm{O} 3-\mathrm{Dy} 1-\mathrm{N} 8$ & $72.64(12)$ & $\mathrm{N} 2-\mathrm{Dy} 1-\mathrm{O} 4$ & $72.18(12)$ \\
\hline
\end{tabular}




\begin{tabular}{|c|c|c|c|}
\hline $\mathrm{O} 4-\mathrm{Dy} 1-\mathrm{N} 1$ & $69.25(14)$ & $\mathrm{N} 2-\mathrm{Dy} 1-\mathrm{N} 1$ & $66.02(12)$ \\
\hline $\mathrm{O} 4-\mathrm{Dy} 1-\mathrm{N} 8$ & $136.69(12)$ & $\mathrm{N} 2-\mathrm{Dy} 1-\mathrm{N} 5$ & $69.61(12)$ \\
\hline $\mathrm{O} 5-\mathrm{Dy} 1-\mathrm{O} 3$ & $134.12(12)$ & $\mathrm{N} 2-\mathrm{Dy} 1-\mathrm{N} 8$ & $133.58(12)$ \\
\hline $\mathrm{O} 5-\mathrm{Dy} 1-\mathrm{O} 4$ & $65.14(12)$ & $\mathrm{N} 5-\mathrm{Dy} 1-\mathrm{O} 4$ & $109.79(12)$ \\
\hline $\mathrm{O} 5-\mathrm{Dy} 1-\mathrm{N} 1$ & $133.64(12)$ & $\mathrm{N} 5-\mathrm{Dy} 1-\mathrm{N} 1$ & $133.40(12)$ \\
\hline $\mathrm{O} 5-\mathrm{Dy} 1-\mathrm{N} 2$ & $106.12(12)$ & N5-Dy $1-\mathrm{N} 8$ & $66.18(11)$ \\
\hline $\mathrm{O} 5-\mathrm{Dy} 1-\mathrm{N} 5$ & $72.23(13)$ & $\mathrm{N} 8-\mathrm{Dy} 1-\mathrm{N} 1$ & $146.54(13)$ \\
\hline \multicolumn{4}{|c|}{3 Symmetry code: (A) $-x+1,-y+1,-z+2 ;$ (B) $-y+4 / 3, x-y+2 / 3, z-1 / 3$. } \\
\hline \multicolumn{4}{|c|}{ Atomic Distances [̊̊] } \\
\hline Dy $1-O 1 B$ & $2.493(10)$ & Dy $1-\mathrm{N} 2$ & $2.400(12)$ \\
\hline $\mathrm{Dy} 1-\mathrm{O} 2$ & $2.243(8)$ & Dy1-N5 & $2.441(12)$ \\
\hline $\mathrm{Dy} 1-\mathrm{O} 2 \mathrm{~A}$ & $2.243(9)$ & Dy1-N6B & $2.455(12)$ \\
\hline Dy $1-\mathrm{N} 1$ & $2.528(13)$ & Dy $1-\mathrm{N} 8$ & $2.698(13)$ \\
\hline \multicolumn{4}{|l|}{ Bond Angles $\left[{ }^{\circ}\right]$} \\
\hline $\mathrm{O} 1 \mathrm{~B}-\mathrm{Dy} 1-\mathrm{N} 1$ & $79.1(4)$ & $\mathrm{O} 2 \mathrm{~A}-\mathrm{Dy} 1-\mathrm{N} 8$ & $80.5(4)$ \\
\hline $\mathrm{O} 1 \mathrm{~B}-\mathrm{Dy} 1-\mathrm{N} 8$ & $77.8(4)$ & $\mathrm{N} 1-\mathrm{Dy} 1-\mathrm{N} 8$ & $154.6(4)$ \\
\hline $\mathrm{O} 2-\mathrm{Dy} 1-\mathrm{O} 1 \mathrm{~B}$ & $143.7(4)$ & N2-Dy1-O1B & $128.5(4)$ \\
\hline $\mathrm{O} 2 \mathrm{~A}-\mathrm{Dy} 1-\mathrm{O} 1 \mathrm{~B}$ & $74.2(4)$ & $\mathrm{N} 2-\mathrm{Dy} 1-\mathrm{N} 1$ & $68.6(5)$ \\
\hline $\mathrm{O} 2-\mathrm{Dy} 1-\mathrm{O} 2 \mathrm{~A}$ & $69.9(4)$ & $\mathrm{N} 2-\mathrm{Dy} 1-\mathrm{N} 5$ & $71.3(4)$ \\
\hline $\mathrm{O} 2-\mathrm{Dy} 1-\mathrm{N} 1$ & $91.7(4)$ & $\mathrm{N} 2-\mathrm{Dy} 1-\mathrm{N} 6 \mathrm{~B}$ & $79.7(4)$ \\
\hline $\mathrm{O} 2 \mathrm{~A}-\mathrm{Dy} 1-\mathrm{N} 1$ & $83.2(4)$ & $\mathrm{N} 2-\mathrm{Dy} 1-\mathrm{N} 8$ & $135.5(4)$ \\
\hline $\mathrm{O} 2-\mathrm{Dy} 1-\mathrm{N} 2$ & $77.8(4)$ & N5-Dy1-O1B & $124.7(4)$ \\
\hline $\mathrm{O} 2 \mathrm{~A}-\mathrm{Dy} 1-\mathrm{N} 2$ & $136.1(4)$ & $\mathrm{N} 5-\mathrm{Dy} 1-\mathrm{N} 1$ & $139.6(5)$ \\
\hline $\mathrm{O} 2-\mathrm{Dy} 1-\mathrm{N} 5$ & $84.4(4)$ & N5-Dy1-N6B & $76.2(4)$ \\
\hline $\mathrm{O} 2 \mathrm{~A}-\mathrm{Dy} 1-\mathrm{N} 5$ & $131.5(4)$ & $\mathrm{N} 5-\mathrm{Dy} 1-\mathrm{N} 8$ & $64.3(4)$ \\
\hline $\mathrm{O} 2-\mathrm{Dy} 1-\mathrm{N} 6 \mathrm{~B}$ & $154.0(4)$ & N6B-Dy $1-O 1 B$ & $62.1(4)$ \\
\hline $\mathrm{O} 2 \mathrm{~A}-\mathrm{Dy} 1-\mathrm{N} 6 \mathrm{~B}$ & $136.1(4)$ & $\mathrm{N} 6 \mathrm{~B}-\mathrm{Dy} 1-\mathrm{N} 1$ & $92.0(4)$ \\
\hline $\mathrm{O} 2-\mathrm{Dy} 1-\mathrm{N} 8$ & $100.7(4)$ & $\mathrm{N} 6 \mathrm{~B}-\mathrm{Dy} 1-\mathrm{N} 8$ & $86.4(4)$ \\
\hline 4 & & & \\
\hline
\end{tabular}




\begin{tabular}{|c|c|c|c|}
\hline \multicolumn{4}{|c|}{ Atomic Distances [Å] } \\
\hline Dy $1-\mathrm{N} 1$ & $2.630(3)$ & Dy $1-O 2$ & $2.492(3)$ \\
\hline Dy $1-\mathrm{N} 2$ & $2.455(3)$ & Dy $1-O 4$ & $2.403(3)$ \\
\hline Dy $1-\mathrm{N} 5$ & $2.442(3)$ & Dy $1-05$ & $2.487(3)$ \\
\hline Dy $1-\mathrm{N} 8$ & $2.600(3)$ & Dy1-O6 & $2.406(3)$ \\
\hline Dy $1-O 1$ & $2.535(3)$ & & \\
\hline \multicolumn{4}{|l|}{ Bond Angles $\left[{ }^{\circ}\right]$} \\
\hline $\mathrm{N} 2-\mathrm{Dy} 1-\mathrm{N} 1$ & $65.76(10)$ & $\mathrm{O} 4-\mathrm{Dy} 1-\mathrm{N} 2$ & $73.98(10)$ \\
\hline $\mathrm{N} 2-\mathrm{Dy} 1-\mathrm{N} 8$ & $132.78(11)$ & $\mathrm{O} 4-\mathrm{Dy} 1-\mathrm{N} 5$ & $106.88(11)$ \\
\hline $\mathrm{N} 2-\mathrm{Dy} 1-\mathrm{O} 1$ & $77.47(11)$ & $\mathrm{O} 4-\mathrm{Dy} 1-\mathrm{N} 8$ & $136.03(10)$ \\
\hline $\mathrm{N} 2-\mathrm{Dy} 1-\mathrm{O} 2$ & $117.78(10)$ & $\mathrm{O} 4-\mathrm{Dy} 1-\mathrm{O} 1$ & $147.39(10)$ \\
\hline $\mathrm{N} 2-\mathrm{Dy} 1-\mathrm{O} 5$ & $116.84(10)$ & $\mathrm{O} 4-\mathrm{Dy} 1-\mathrm{O} 2$ & $134.66(10)$ \\
\hline N5-Dy1-N1 & $131.99(10)$ & $\mathrm{O} 4-\mathrm{Dy} 1-\mathrm{O} 5$ & $69.20(10)$ \\
\hline $\mathrm{N} 5-\mathrm{Dy} 1-\mathrm{N} 2$ & $69.87(10)$ & O4-Dy1-O6 & $75.21(10)$ \\
\hline $\mathrm{N} 5-\mathrm{Dy} 1-\mathrm{N} 8$ & $66.59(10)$ & $\mathrm{O} 5-\mathrm{Dy} 1-\mathrm{N} 1$ & $143.56(10)$ \\
\hline N5-Dy1-O1 & 77.08 (11) & $\mathrm{O} 5-\mathrm{Dy} 1-\mathrm{N} 8$ & $67.25(10)$ \\
\hline $\mathrm{N} 5-\mathrm{Dy} 1-\mathrm{O} 2$ & $118.34(10)$ & $\mathrm{O} 5-\mathrm{Dy} 1-\mathrm{O} 1$ & $139.87(11)$ \\
\hline N5-Dy1-O5 & $74.19(10)$ & $\mathrm{O} 5-\mathrm{Dy} 1-\mathrm{O} 2$ & $124.77(10)$ \\
\hline $\mathrm{N} 8-\mathrm{Dy} 1-\mathrm{N} 1$ & $140.54(11)$ & $\mathrm{O} 6-\mathrm{Dy} 1-\mathrm{N} 1$ & $80.83(10)$ \\
\hline $\mathrm{O} 1-\mathrm{Dy} 1-\mathrm{N} 1$ & $76.37(11)$ & O6-Dy $1-\mathrm{N} 2$ & $138.32(10)$ \\
\hline $\mathrm{O} 1-\mathrm{Dy} 1-\mathrm{N} 8$ & $75.92(11)$ & O6-Dy $1-\mathrm{N} 5$ & $147.16(10)$ \\
\hline $\mathrm{O} 2-\mathrm{Dy} 1-\mathrm{N} 1$ & $70.21(10)$ & O6-Dy $1-\mathrm{N} 8$ & $88.86(11)$ \\
\hline $\mathrm{O} 2-\mathrm{Dy} 1-\mathrm{N} 8$ & $70.54(10)$ & O6-Dy1-O1 & $119.30(11)$ \\
\hline $\mathrm{O} 2-\mathrm{Dy} 1-\mathrm{O} 1$ & $50.61(10)$ & $\mathrm{O} 6-\mathrm{Dy} 1-\mathrm{O} 2$ & $68.80(10)$ \\
\hline $\mathrm{O} 4-\mathrm{Dy} 1-\mathrm{N} 1$ & $77.77(11)$ & O6-Dy1-O5 & $76.32(10)$ \\
\hline \multicolumn{4}{|c|}{5} \\
\hline \multicolumn{4}{|c|}{ Atomic Distances $[\AA ̊]$} \\
\hline Dy $1-\mathrm{N} 1$ & $2.496(4)$ & Dy2-N12 & $2.521(4)$ \\
\hline Dy $1-\mathrm{N} 2$ & $2.433(4)$ & Dy2-N13 & $2.440(4)$ \\
\hline
\end{tabular}




\begin{tabular}{llll}
\hline Dy1-N5 & $2.450(4)$ & Dy2-N16 & $2.453(4)$ \\
Dy1-N8 & $2.540(4)$ & Dy2-N19 & $2.544(4)$ \\
Dy1-O3 & $2.408(4)$ & Dy2-O12 & $2.508(4)$ \\
Dy1-O4 & $2.431(4)$ & Dy2-O13 & $2.456(3)$ \\
Dy1-O6 & $2.441(4)$ & Dy2-O15 & $2.409(4)$ \\
Dy1-O7 & $2.497(4)$ & Dy2-O16 & $2.421(4)$ \\
Dy1-O09 & $2.391(3)$ & Dy2-O18 & $2.381(3)$
\end{tabular}

Bond Angles $\left[{ }^{\circ}\right]$

\begin{tabular}{|c|c|c|c|}
\hline $\mathrm{N} 1-\mathrm{Dy} 1-\mathrm{N} 8$ & $151.39(14)$ & N12-Dy2-N19 & $152.56(14)$ \\
\hline $\mathrm{N} 1-\mathrm{Dy} 1-\mathrm{O} 7$ & $70.16(12)$ & N13-Dy2-N12 & $67.14(13)$ \\
\hline $\mathrm{N} 2-\mathrm{Dy} 1-\mathrm{N} 1$ & $67.19(13)$ & N13-Dy2-N16 & $69.39(13)$ \\
\hline $\mathrm{N} 2-\mathrm{Dy} 1-\mathrm{N} 5$ & $70.25(13)$ & N13-Dy2-N19 & $135.15(14)$ \\
\hline $\mathrm{N} 2-\mathrm{Dy} 1-\mathrm{N} 8$ & $135.70(14)$ & $\mathrm{N} 13-\mathrm{Dy} 2-\mathrm{O} 12$ & $72.39(14)$ \\
\hline $\mathrm{N} 2-\mathrm{Dy} 1-\mathrm{O} 6$ & $95.44(13)$ & $\mathrm{N} 13-\mathrm{Dy} 2-\mathrm{O} 13$ & $100.77(13)$ \\
\hline $\mathrm{N} 2-\mathrm{Dy} 1-\mathrm{O} 7$ & $75.49(13)$ & N16-Dy2-N12 & $135.74(13)$ \\
\hline $\mathrm{N} 5-\mathrm{Dy} 1-\mathrm{N} 1$ & 136.07 (13) & N16-Dy2-N19 & $66.53(13)$ \\
\hline N5-Dy1-N8 & $66.07(14)$ & $\mathrm{N} 16-\mathrm{Dy} 2-\mathrm{O} 12$ & $102.41(13)$ \\
\hline N5-Dy1-O7 & $109.13(13)$ & $\mathrm{N} 16-\mathrm{Dy} 2-\mathrm{O} 13$ & $74.14(13)$ \\
\hline $\mathrm{O} 3-\mathrm{Dy} 1-\mathrm{N} 1$ & $82.49(15)$ & $\mathrm{O} 12-\mathrm{Dy} 2-\mathrm{N} 12$ & $72.04(14)$ \\
\hline $\mathrm{O} 3-\mathrm{Dy} 1-\mathrm{N} 2$ & $76.79(14)$ & O12-Dy2-N19 & $124.92(15)$ \\
\hline $\mathrm{O} 3-\mathrm{Dy} 1-\mathrm{N} 5$ & $77.76(14)$ & $\mathrm{O} 13-\mathrm{Dy} 2-\mathrm{N} 12$ & $121.88(13)$ \\
\hline $\mathrm{O} 3-\mathrm{Dy} 1-\mathrm{N} 8$ & $87.04(15)$ & O13-Dy2-N19 & $74.94(14)$ \\
\hline $\mathrm{O} 3-\mathrm{Dy} 1-\mathrm{O} 4$ & $52.53(13)$ & $\mathrm{O} 13-\mathrm{Dy} 2-\mathrm{O} 12$ & $50.88(12)$ \\
\hline O3-Dy1-O6 & $150.32(14)$ & $\mathrm{O} 15-\mathrm{Dy} 2-\mathrm{N} 12$ & $86.88(15)$ \\
\hline $\mathrm{O} 3-\mathrm{Dy} 1-\mathrm{O} 7$ & $146.81(13)$ & $\mathrm{O} 15-\mathrm{Dy} 2-\mathrm{N} 13$ & $80.62(14)$ \\
\hline $\mathrm{O} 4-\mathrm{Dy} 1-\mathrm{N} 1$ & 75.79 (13) & O15-Dy2-N16 & $78.23(14)$ \\
\hline $\mathrm{O} 4-\mathrm{Dy} 1-\mathrm{N} 2$ & $120.13(13)$ & O15-Dy2-N19 & $82.84(15)$ \\
\hline $\mathrm{O} 4-\mathrm{Dy} 1-\mathrm{N} 5$ & $118.63(14)$ & $\mathrm{O} 15-\mathrm{Dy} 2-\mathrm{O} 12$ & $150.52(14)$ \\
\hline $\mathrm{O} 4-\mathrm{Dy} 1-\mathrm{N} 8$ & $76.71(14)$ & O15-Dy2-O13 & $149.68(13)$ \\
\hline
\end{tabular}




\begin{tabular}{|c|c|c|c|}
\hline O4-Dy1-O6 & $144.39(13)$ & $\mathrm{O} 15-\mathrm{Dy} 2-\mathrm{O} 16$ & $53.21(14)$ \\
\hline $\mathrm{O} 4-\mathrm{Dy} 1-\mathrm{O} 7$ & $132.24(12)$ & $\mathrm{O} 16-\mathrm{Dy} 2-\mathrm{N} 12$ & $77.06(13)$ \\
\hline O6-Dy1-N1 & $121.31(14)$ & $\mathrm{O} 16-\mathrm{Dy} 2-\mathrm{N} 13$ & $122.50(14)$ \\
\hline O6-Dy1-N5 & $72.68(14)$ & $\mathrm{O} 16-\mathrm{Dy} 2-\mathrm{N} 16$ & $121.74(13)$ \\
\hline O6-Dy1-N8 & $78.71(14)$ & $\mathrm{O} 16-\mathrm{Dy} 2-\mathrm{N} 19$ & $76.41(14)$ \\
\hline O6-Dy1-O7 & $51.15(12)$ & $\mathrm{O} 16-\mathrm{Dy} 2-\mathrm{O} 12$ & $135.82(13)$ \\
\hline $\mathrm{O} 7-\mathrm{Dy} 1-\mathrm{N} 8$ & $125.81(13)$ & $\mathrm{O} 16-\mathrm{Dy} 2-\mathrm{O} 13$ & $136.58(12)$ \\
\hline O9-Dy1-N1 & $83.30(12)$ & $\mathrm{O} 18-\mathrm{Dy} 2-\mathrm{N} 12$ & $83.48(13)$ \\
\hline O9-Dy1-N2 & $141.37(14)$ & $\mathrm{O} 18-\mathrm{Dy} 2-\mathrm{N} 13$ & $140.50(13)$ \\
\hline O9-Dy1-N5 & $139.64(12)$ & $\mathrm{O} 18-\mathrm{Dy} 2-\mathrm{N} 16$ & $138.57(14)$ \\
\hline O9-Dy1-N8 & 81.09 (14) & $\mathrm{O} 18-\mathrm{Dy} 2-\mathrm{N} 19$ & $81.82(13)$ \\
\hline O9-Dy1-O3 & $124.97(13)$ & $\mathrm{O} 18-\mathrm{Dy} 2-\mathrm{O} 12$ & $73.81(13)$ \\
\hline O9-Dy1-O4 & $72.46(13)$ & $\mathrm{O} 18-\mathrm{Dy} 2-\mathrm{O} 13$ & $72.23(13)$ \\
\hline O9-Dy1-O6 & $78.62(13)$ & $\mathrm{O} 18-\mathrm{Dy} 2-\mathrm{O} 15$ & $125.10(14)$ \\
\hline O9-Dy1-O7 & $71.21(12)$ & $\mathrm{O} 18-\mathrm{Dy} 2-\mathrm{O} 16$ & $71.97(14)$ \\
\hline \multicolumn{4}{|l|}{6} \\
\hline \multicolumn{4}{|c|}{ Atomic Distances $[\AA]]$} \\
\hline Dy $1-\mathrm{N} 1$ & $2.579(11)$ & $\mathrm{Dy} 1-\mathrm{O} 2$ & $2.488(10)$ \\
\hline Dy $1-\mathrm{N} 2$ & $2.445(10)$ & Dy $1-O 4$ & $2.423(10)$ \\
\hline Dy $1-\mathrm{N} 5$ & $2.516(10)$ & Dy $1-O 5$ & $2.461(11)$ \\
\hline Dy $1-\mathrm{N} 8$ & $2.557(11)$ & Dy $1-07$ & $2.489(13)$ \\
\hline Dy1-O1 & $2.506(11)$ & Dy $1-08$ & $2.490(12)$ \\
\hline \multicolumn{4}{|l|}{ Bond Angles $\left[^{\circ}\right]$} \\
\hline $\mathrm{N} 2-\mathrm{Dy} 1-\mathrm{N} 1$ & $65.9(4)$ & $\mathrm{O} 4-\mathrm{Dy} 1-\mathrm{O} 5$ & $52.9(4)$ \\
\hline $\mathrm{N} 2-\mathrm{Dy} 1-\mathrm{N} 5$ & $68.8(3)$ & $\mathrm{O} 4-\mathrm{Dy} 1-\mathrm{O} 7$ & $144.0(5)$ \\
\hline $\mathrm{N} 2-\mathrm{Dy} 1-\mathrm{N} 8$ & $133.3(4)$ & $\mathrm{O} 4-\mathrm{Dy} 1-\mathrm{O} 8$ & $115.5(4)$ \\
\hline $\mathrm{N} 2-\mathrm{Dy} 1-\mathrm{O} 1$ & $82.8(5)$ & $\mathrm{O} 5-\mathrm{Dy} 1-\mathrm{N} 1$ & $75.1(4)$ \\
\hline $\mathrm{N} 2-\mathrm{Dy} 1-\mathrm{O} 2$ & $73.6(3)$ & O5-Dy1-N5 & $118.0(3)$ \\
\hline $\mathrm{N} 2-\mathrm{Dy} 1-\mathrm{O} 5$ & $117.9(4)$ & $\mathrm{O} 5-\mathrm{Dy} 1-\mathrm{N} 8$ & $77.4(4)$ \\
\hline
\end{tabular}




\begin{tabular}{|c|c|c|c|}
\hline $\mathrm{N} 2-\mathrm{Dy} 1-\mathrm{O} 7$ & $137.6(4)$ & O5-Dy $1-O 1$ & $159.2(5)$ \\
\hline $\mathrm{N} 2-\mathrm{Dy} 1-\mathrm{O} 8$ & $137.5(4)$ & $\mathrm{O} 5-\mathrm{Dy} 1-\mathrm{O} 2$ & $130.2(4)$ \\
\hline $\mathrm{N} 5-\mathrm{Dy} 1-\mathrm{N} 1$ & $133.1(3)$ & $\mathrm{O} 5-\mathrm{Dy} 1-\mathrm{O} 7$ & $102.0(4)$ \\
\hline $\mathrm{N} 5-\mathrm{Dy} 1-\mathrm{N} 8$ & $65.5(3)$ & $\mathrm{O} 5-\mathrm{Dy} 1-\mathrm{O} 8$ & $62.8(4)$ \\
\hline $\mathrm{N} 8-\mathrm{Dy} 1-\mathrm{N} 1$ & $152.2(4)$ & O7-Dy $1-\mathrm{N} 1$ & $115.3(4)$ \\
\hline $\mathrm{O} 1-\mathrm{Dy} 1-\mathrm{N} 1$ & $114.9(4)$ & O7-Dy1-N5 & $105.9(3)$ \\
\hline $\mathrm{O} 1-\mathrm{Dy} 1-\mathrm{N} 5$ & $69.6(4)$ & $\mathrm{O} 7-\mathrm{Dy} 1-\mathrm{N} 8$ & $66.5(4)$ \\
\hline $\mathrm{O} 2-\mathrm{Dy} 1-\mathrm{O} 8$ & $77.0(4)$ & $\mathrm{O} 7-\mathrm{Dy} 1-\mathrm{O} 1$ & $57.5(5)$ \\
\hline $\mathrm{O} 4-\mathrm{Dy} 1-\mathrm{N} 1$ & $85.3(4)$ & $\mathrm{O} 7-\mathrm{Dy} 1-\mathrm{O} 8$ & $50.8(3)$ \\
\hline $\mathrm{O} 4-\mathrm{Dy} 1-\mathrm{N} 2$ & $76.9(4)$ & $\mathrm{O} 8-\mathrm{Dy} 1-\mathrm{N} 1$ & $74.6(4)$ \\
\hline $\mathrm{O} 4-\mathrm{Dy} 1-\mathrm{N} 5$ & $73.3(4)$ & $\mathrm{O} 8-\mathrm{Dy} 1-\mathrm{N} 5$ & $152.3(4)$ \\
\hline $\mathrm{O} 4-\mathrm{Dy} 1-\mathrm{N} 8$ & $81.7(4)$ & $\mathrm{O} 8-\mathrm{Dy} 1-\mathrm{N} 8$ & $89.1(4)$ \\
\hline $\mathrm{O} 4-\mathrm{Dy} 1-\mathrm{O} 1$ & $142.1(4)$ & $\mathrm{O} 8-\mathrm{Dy} 1-\mathrm{O} 1$ & $101.1(4)$ \\
\hline $\mathrm{O} 4-\mathrm{Dy} 1-\mathrm{O} 2$ & $145.4(3)$ & & \\
\hline \multicolumn{4}{|c|}{7 Symmetry code: (A) -x, -y, -z. } \\
\hline \multicolumn{4}{|c|}{ Atomic Distances $[\AA ̊]$} \\
\hline Dy $1-\mathrm{N} 1$ & $2.603(3)$ & Dy2-N6 & $2.431(3)$ \\
\hline Dy $1-\mathrm{N} 2$ & $2.474(3)$ & Dy $2-\mathrm{N} 8$ & $2.658(3)$ \\
\hline Dy $1-\mathrm{N} 5$ & $2.512(3)$ & $\mathrm{Dy} 2-\mathrm{O} 2 \mathrm{~A}$ & $2.293(2)$ \\
\hline Dy $1-\mathrm{O} 1$ & $2.331(2)$ & Dy2-O3A & $2.470(2)$ \\
\hline Dy $1-O 3$ & $2.708(2)$ & Dy2-O6 & $2.413(2)$ \\
\hline Dy1-O4 & $2.419(3)$ & Dy2-O7A & $2.353(2)$ \\
\hline Dy $1-05$ & $2.363(2)$ & Dy2-O7 & $2.415(2)$ \\
\hline Dy $1-06$ & $2.486(2)$ & Dy2-O8 & $2.459(2)$ \\
\hline Dy $1-\mathrm{O} 7$ & $2.330(2)$ & Dy2-O9 & $2.537(2)$ \\
\hline \multicolumn{4}{|l|}{ Bond Angles $\left[{ }^{\circ}\right]$} \\
\hline $\mathrm{N} 1-\mathrm{Dy} 1-\mathrm{O} 3$ & $77.71(8)$ & $\mathrm{N} 6-\mathrm{Dy} 2-\mathrm{O} 3 \mathrm{~A}$ & $137.75(8)$ \\
\hline $\mathrm{N} 2-\mathrm{Dy} 1-\mathrm{N} 1$ & $65.52(8)$ & $\mathrm{N} 6-\mathrm{Dy} 2-\mathrm{O} 8$ & $95.74(9)$ \\
\hline $\mathrm{N} 2-\mathrm{Dy} 1-\mathrm{N} 5$ & $67.77(8)$ & N6-Dy2-O9 & $134.67(8)$ \\
\hline
\end{tabular}




\begin{tabular}{|c|c|c|c|}
\hline $\mathrm{N} 2-\mathrm{Dy} 1-\mathrm{O} 3$ & $117.62(8)$ & $\mathrm{O} 2 \mathrm{~A}-\mathrm{Dy} 2-\mathrm{N} 6$ & $80.59(9)$ \\
\hline N2-Dy1-O6 & $123.79(8)$ & $\mathrm{O} 2 \mathrm{~A}-\mathrm{Dy} 2-\mathrm{N} 8$ & $73.75(9)$ \\
\hline N5-Dy1-N1 & $133.15(8)$ & $\mathrm{O} 2 \mathrm{~A}-\mathrm{Dy} 2-\mathrm{N} 9$ & $151.61(8)$ \\
\hline N5-Dy1-O3 & $129.21(8)$ & $\mathrm{O} 2 \mathrm{~A}-\mathrm{Dy} 2-\mathrm{O} 3 \mathrm{~A}$ & $76.07(8)$ \\
\hline $\mathrm{O} 1-\mathrm{Dy} 1-\mathrm{N} 1$ & $73.14(8)$ & $\mathrm{O} 2 \mathrm{~A}-\mathrm{Dy} 2-\mathrm{O} 6$ & $138.09(8)$ \\
\hline $\mathrm{O} 1-\mathrm{Dy} 1-\mathrm{N} 2$ & $133.80(8)$ & $\mathrm{O} 2 \mathrm{~A}-\mathrm{Dy} 2-\mathrm{O} 7$ & $72.66(8)$ \\
\hline O1-Dy1-N5 & $145.75(9)$ & $\mathrm{O} 2 \mathrm{~A}-\mathrm{Dy} 2-\mathrm{O} 7 \mathrm{~A}$ & $81.78(8)$ \\
\hline $\mathrm{O} 1-\mathrm{Dy} 1-\mathrm{O} 3$ & $70.24(8)$ & $\mathrm{O} 2 \mathrm{~A}-\mathrm{Dy} 2-\mathrm{O} 8$ & $139.40(9)$ \\
\hline $\mathrm{O} 1-\mathrm{Dy} 1-\mathrm{O} 4$ & $115.14(9)$ & $\mathrm{O} 2 \mathrm{~A}-\mathrm{Dy} 2-\mathrm{O} 9$ & $144.62(8)$ \\
\hline O1-Dy1-O5 & $77.76(9)$ & $\mathrm{O} 3 \mathrm{~A}-\mathrm{Dy} 2-\mathrm{N} 8$ & $76.21(8)$ \\
\hline O1-Dy1-O6 & $72.54(8)$ & $\mathrm{O} 3 \mathrm{~A}-\mathrm{Dy} 2-\mathrm{O} 9$ & $73.56(8)$ \\
\hline O4-Dy1-N1 & $72.44(9)$ & O6-Dy2-N6 & $69.72(8)$ \\
\hline $\mathrm{O} 4-\mathrm{Dy} 1-\mathrm{N} 2$ & $71.33(8)$ & O6-Dy2-N8 & $115.17(8)$ \\
\hline $\mathrm{O} 4-\mathrm{Dy} 1-\mathrm{N} 5$ & $96.08(9)$ & $\mathrm{O} 6-\mathrm{Dy} 2-\mathrm{O} 3 \mathrm{~A}$ & $144.99(8)$ \\
\hline $\mathrm{O} 4-\mathrm{Dy} 1-\mathrm{O} 3$ & $49.76(8)$ & O6-Dy2-O7 & $70.00(7)$ \\
\hline O4-Dy1-O6 & $152.76(8)$ & $\mathrm{O} 6-\mathrm{Dy} 2-\mathrm{O} 8$ & $74.27(8)$ \\
\hline O5-Dy1-N1 & $88.58(8)$ & O6-Dy2-O9 & $71.73(8)$ \\
\hline O5-Dy1-N2 & $81.41(9)$ & $\mathrm{O} 7-\mathrm{Dy} 2-\mathrm{N} 6$ & $70.66(8)$ \\
\hline O5-Dy1-N5 & $81.18(9)$ & $\mathrm{O} 7 \mathrm{~A}-\mathrm{Dy} 2-\mathrm{N} 6$ & $140.03(8)$ \\
\hline O5-Dy1-O3 & $147.61(9)$ & $\mathrm{O} 7 \mathrm{~A}-\mathrm{Dy} 2-\mathrm{N} 8$ & $142.25(8)$ \\
\hline $\mathrm{O} 5-\mathrm{Dy} 1-\mathrm{O} 4$ & $151.35(8)$ & $\mathrm{O} 7-\mathrm{Dy} 2-\mathrm{N} 8$ & $126.41(8)$ \\
\hline O5-Dy1-O6 & $53.47(7)$ & $\mathrm{O} 7 \mathrm{~A}-\mathrm{Dy} 2-\mathrm{O} 3 \mathrm{~A}$ & $70.20(7)$ \\
\hline O6-Dy1-N1 & $133.07(8)$ & $\mathrm{O} 7-\mathrm{Dy} 2-\mathrm{O} 3 \mathrm{~A}$ & $131.90(8)$ \\
\hline O6-Dy1-N5 & $73.23(8)$ & $\mathrm{O} 7 \mathrm{~A}-\mathrm{Dy} 2-\mathrm{O} 6$ & $102.28(8)$ \\
\hline O6-Dy1-O3 & $118.27(7)$ & $\mathrm{O} 7 \mathrm{~A}-\mathrm{Dy} 2-\mathrm{O} 7$ & $69.91(8)$ \\
\hline O7-Dy1-N1 & $144.04(8)$ & $\mathrm{O} 7-\mathrm{Dy} 2-\mathrm{O} 8$ & $144.24(8)$ \\
\hline $\mathrm{O} 7-\mathrm{Dy} 1-\mathrm{N} 2$ & $130.52(8)$ & $\mathrm{O} 7 \mathrm{~A}-\mathrm{Dy} 2-\mathrm{O} 8$ & $120.44(8)$ \\
\hline O7-Dy1-N5 & $74.09(8)$ & O7-Dy2-O9 & $116.45(8)$ \\
\hline $\mathrm{O} 7-\mathrm{Dy} 1-\mathrm{O} 1$ & $95.19(8)$ & $\mathrm{O} 7 \mathrm{~A}-\mathrm{Dy} 2-\mathrm{O} 9$ & $71.21(8)$ \\
\hline
\end{tabular}




\begin{tabular}{llll}
\hline O7-Dy1-O3 & $66.36(7)$ & O8-Dy2-N8 & $68.69(8)$ \\
O7-Dy1-O4 & $82.94(8)$ & O8-Dy2-N9 & $25.52(8)$ \\
O7-Dy1-O5 & $122.82(8)$ & O8-Dy2-O3A & $80.53(8)$ \\
O7-Dy1-O6 & $70.11(7)$ & O8-Dy2-O9 & $50.84(8)$ \\
N6-Dy2-N8 & $63.58(8)$ & O9-Dy2-N8 & $115.24(8)$
\end{tabular}

8 Symmetry code: (A) $-x+1,-y+1,-z+1$.

Atomic Distances [̊̊]

$\begin{array}{llll}\text { Dy1-N1 } & 2.618(5) & \text { Dy2-N6 } & 2.433(5) \\ \text { Dy1-N2 } & 2.502(5) & \text { Dy2-N8 } & 2.664(6) \\ \text { Dy1-N5 } & 2.505(5) & \text { Dy2-O2 } & 2.416(4) \\ \text { Dy1-O1 } & 2.368(5) & \text { Dy2-O3A } & 2.349(4) \\ \text { Dy1-O2 } & 2.505(4) & \text { Dy2-O3 } & 2.418(4) \\ \text { Dy1-O3 } & 2.344(4) & \text { Dy2-O4 } & 2.294(5) \\ \text { Dy1-O5A } & 2.344(5) & \text { Dy2-O6 } & 2.467(5) \\ \text { Dy1-O9A } & 2.660(5) & \text { Dy2-O7 } & 2.534(5) \\ \text { Dy1-O10A } & 2.445(5) & \text { Dy2-O9 } & 2.511(5)\end{array}$

Bond Angles $\left[{ }^{\circ}\right]$

\begin{tabular}{|c|c|c|c|}
\hline N1-Dy1-O9A & $77.03(16)$ & N6-Dy2-N8 & $63.68(18)$ \\
\hline $\mathrm{N} 2-\mathrm{Dy} 1-\mathrm{N} 1$ & $65.02(17)$ & N6-Dy2-O6 & 96.59 (19) \\
\hline $\mathrm{N} 2-\mathrm{Dy} 1-\mathrm{N} 5$ & $68.20(18)$ & N6-Dy2-O7 & $135.25(18)$ \\
\hline $\mathrm{N} 2-\mathrm{Dy} 1-\mathrm{O} 2$ & $123.59(16)$ & N6-Dy2-O9 & $137.81(16)$ \\
\hline $\mathrm{N} 2-\mathrm{Dy} 1-\mathrm{O} 9 \mathrm{~A}$ & $117.67(16)$ & $\mathrm{O} 2-\mathrm{Dy} 2-\mathrm{N} 6$ & $69.72(17)$ \\
\hline $\mathrm{N} 5-\mathrm{Dy} 1-\mathrm{N} 1$ & $133.17(17)$ & $\mathrm{O} 2-\mathrm{Dy} 2-\mathrm{N} 8$ & $115.08(17)$ \\
\hline N5-Dy1-O9A & $129.06(18)$ & $\mathrm{O} 2-\mathrm{Dy} 2-\mathrm{O} 3$ & $70.12(15)$ \\
\hline $\mathrm{O} 1-\mathrm{Dy} 1-\mathrm{N} 1$ & $89.40(18)$ & $\mathrm{O} 2-\mathrm{Dy} 2-\mathrm{O} 6$ & $75.59(16)$ \\
\hline $\mathrm{O} 1-\mathrm{Dy} 1-\mathrm{N} 2$ & $81.02(18)$ & $\mathrm{O} 2-\mathrm{Dy} 2-\mathrm{O} 7$ & $72.53(16)$ \\
\hline $\mathrm{O} 1-\mathrm{Dy} 1-\mathrm{N} 5$ & $81.4(2)$ & $\mathrm{O} 2-\mathrm{Dy} 2-\mathrm{O} 9$ & $145.75(15)$ \\
\hline $\mathrm{O} 1-\mathrm{Dy} 1-\mathrm{O} 2$ & $53.38(15)$ & $\mathrm{O} 3-\mathrm{Dy} 2-\mathrm{N} 6$ & $70.35(16)$ \\
\hline $\mathrm{O} 1-\mathrm{Dy} 1-\mathrm{O} 9 \mathrm{~A}$ & $147.62(17)$ & $\mathrm{O} 3 \mathrm{~A}-\mathrm{Dy} 2-\mathrm{N} 6$ & $139.66(17)$ \\
\hline
\end{tabular}




\begin{tabular}{|c|c|c|c|}
\hline O1-Dy1-O10A & $151.44(17)$ & $\mathrm{O} 3-\mathrm{Dy} 2-\mathrm{N} 8$ & $126.23(16)$ \\
\hline $\mathrm{O} 2-\mathrm{Dy} 1-\mathrm{N} 1$ & $133.85(16)$ & $\mathrm{O} 3 \mathrm{~A}-\mathrm{Dy} 2-\mathrm{N} 8$ & $142.23(16)$ \\
\hline $\mathrm{O} 2-\mathrm{Dy} 1-\mathrm{N} 5$ & $73.24(18)$ & $\mathrm{O} 3 \mathrm{~A}-\mathrm{Dy} 2-\mathrm{O} 2$ & $102.42(15)$ \\
\hline O2-Dy1-O9A & $118.48(14)$ & $\mathrm{O} 3 \mathrm{~A}-\mathrm{Dy} 2-\mathrm{O} 3$ & $69.88(16)$ \\
\hline $\mathrm{O} 3-\mathrm{Dy} 1-\mathrm{N} 1$ & $144.06(17)$ & $\mathrm{O} 3-\mathrm{Dy} 2-\mathrm{O} 6$ & $145.67(16)$ \\
\hline $\mathrm{O} 3-\mathrm{Dy} 1-\mathrm{N} 2$ & $130.82(17)$ & $\mathrm{O} 3 \mathrm{~A}-\mathrm{Dy} 2-\mathrm{O} 6$ & $120.40(17)$ \\
\hline $\mathrm{O} 3-\mathrm{Dy} 1-\mathrm{N} 5$ & $73.37(16)$ & $\mathrm{O} 3 \mathrm{~A}-\mathrm{Dy} 2-\mathrm{O} 7$ & $71.55(16)$ \\
\hline $\mathrm{O} 3-\mathrm{Dy} 1-\mathrm{O} 1$ & $122.34(16)$ & $\mathrm{O} 3-\mathrm{Dy} 2-\mathrm{O} 7$ & $117.41(16)$ \\
\hline $\mathrm{O} 3-\mathrm{Dy} 1-\mathrm{O} 2$ & $69.80(14)$ & $\mathrm{O} 3 \mathrm{~A}-\mathrm{Dy} 2-\mathrm{O} 9$ & $69.63(15)$ \\
\hline O3-Dy1-O5A & $96.06(16)$ & O3-Dy2-O9 & $130.92(15)$ \\
\hline O3-Dy1-O9A & $67.09(14)$ & $\mathrm{O} 4-\mathrm{Dy} 2-\mathrm{N} 6$ & $81.26(18)$ \\
\hline O3-Dy1-O10A & $82.85(16)$ & $\mathrm{O} 4-\mathrm{Dy} 2-\mathrm{N} 8$ & $73.72(18)$ \\
\hline $\mathrm{O} 5 \mathrm{~A}-\mathrm{Dy} 1-\mathrm{N} 1$ & $73.17(16)$ & $\mathrm{O} 4-\mathrm{Dy} 2-\mathrm{O} 2$ & $138.97(16)$ \\
\hline $\mathrm{O} 5 \mathrm{~A}-\mathrm{Dy} 1-\mathrm{N} 2$ & $132.74(17)$ & $\mathrm{O} 4-\mathrm{Dy} 2-\mathrm{O} 3 \mathrm{~A}$ & $81.19(17)$ \\
\hline $\mathrm{O} 5 \mathrm{~A}-\mathrm{Dy} 1-\mathrm{N} 5$ & $145.95(19)$ & $\mathrm{O} 4-\mathrm{Dy} 2-\mathrm{O} 3$ & $73.18(15)$ \\
\hline $\mathrm{O} 5 \mathrm{~A}-\mathrm{Dy} 1-\mathrm{O} 1$ & $77.42(18)$ & $\mathrm{O} 4-\mathrm{Dy} 2-\mathrm{O} 6$ & $137.88(17)$ \\
\hline $\mathrm{O} 5 \mathrm{~A}-\mathrm{Dy} 1-\mathrm{O} 2$ & $72.73(16)$ & $\mathrm{O} 4-\mathrm{Dy} 2-\mathrm{O} 7$ & $143.27(16)$ \\
\hline $\mathrm{O} 5 \mathrm{~A}-\mathrm{Dy} 1-\mathrm{O} 9 \mathrm{~A}$ & $70.57(16)$ & $\mathrm{O} 4-\mathrm{Dy} 2-\mathrm{O} 9$ & $74.30(16)$ \\
\hline O5A-Dy1-O10A & $116.00(17)$ & $\mathrm{O} 6-\mathrm{Dy} 2-\mathrm{N} 8$ & $67.98(18)$ \\
\hline $\mathrm{O} 10 \mathrm{~A}-\mathrm{Dy} 1-\mathrm{N} 1$ & $72.31(18)$ & O6-Dy2-O7 & $50.64(18)$ \\
\hline $\mathrm{O} 10 \mathrm{~A}-\mathrm{D} 1 \mathrm{1}-\mathrm{N} 2$ & $71.49(17)$ & O6-Dy2-O9 & $80.29(17)$ \\
\hline O10A-Dy1—N5 & $95.1(2)$ & $\mathrm{O} 7-\mathrm{Dy} 2-\mathrm{N} 8$ & $114.58(17)$ \\
\hline $\mathrm{O} 10 \mathrm{~A}-\mathrm{Dy} 1-\mathrm{O} 2$ & $152.28(16)$ & $\mathrm{O} 9-\mathrm{Dy} 2-\mathrm{N} 8$ & $76.58(16)$ \\
\hline O10A—Dy1—O9A & $50.15(16)$ & O9-Dy2-O7 & $73.40(16)$ \\
\hline
\end{tabular}

